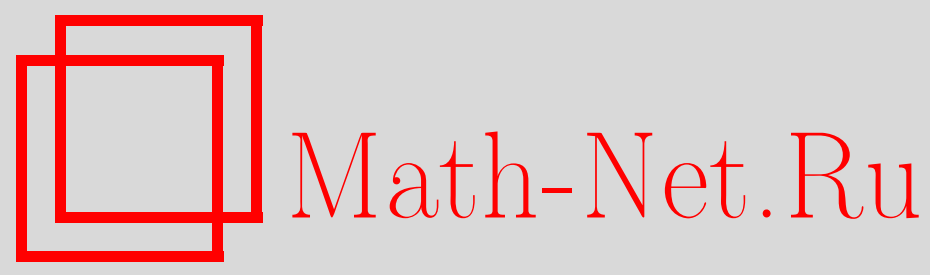

В. Ю. Попов, Об эквациональных теориях многообразий антикоммутативных колец, Матем. заметки, 1999, том 65, выпуск 2, 230-245

DOI: https://doi.org/10.4213/mzm1045

Использование Общероссийского математического портала Math-Net.Ru подразумевает, что вы прочитали и согласны с пользовательским соглашением http://www . mathnet.ru/rus/agreement

Параметры загрузки:

IP: 54.174 .149 .18

26 апреля 2023 г., 06:55:36 


\title{
ОБ ЭКВАЦИОНАЛЬНЫХ ТЕОРИЯХ МНОГООБРАЗИЙ АНТИКОММУТАТИВНЫХ КОЛЕЦ
}

\author{
В. Ю. Попов
}

Доказано существование конечно базируемого многообразия антикоммутативных (в смысле тождества $x^{2}=0$ ) колец с неразрешимой эквациональной теорией.

Библиограффия: 9 названий.

Первьй пример многообразия универсальных алгебр с неразрешимой эквациональной теорией был построен Тарским в 1943-44 годах и опубликован в 1953 году (см. [1]-[3]). Как замечено в [4], всякий пример конечно заданной универсальной алгебры с неразрешимой проблемой равенства слов легко можно модифицировать в пример конечно базируемого многообразия универсальных алгебр с неразрешимой эквациональной теорией. В работе [5] А. И. Мальцев построил конечно базируемое многообразие квазигруп с неразрешимой эквациональной теорией и поставил опубликованный в сборнике [6] вопрос о существовании конечно базируемых многообразий полугрупп, групп и колец (ассоциативных, лиевых) с неразрешимой эквациональной теорией. Положительный ответ на этот вопрос для полугрупп получен в [7], а для групп - в [8]. В работе [9] доказано, что существуют конечно базируемые многообразия метабелевых и коммутативных колец с неразрешимой эквациональной теорией. Многообразия, построенные в работе [9], являются многообразиями колец характеристики 2. Очевидно, что из существования конечно базируемого многообразия коммутативных колец характеристики 2 с неразрешимой эквациональной теорией немедленно вытекает существование конечно базируемого многообразия антикоммутативных (в смысле тождества $x y=-y x$ ) колец характеристики 2 с неразрешимой эквациональной теорией. Однако, из результатов работы [9] не следует сушествование конечно базируемого многообразия антикоммутативных (в смысле тождества $x^{2}=0$ ) колец с неразрешимой эквациональной теорией. Пример подобного многообразия дает следующая

Теорема. Существует конечно базируемое многообразие колеи, удовлетворяющее тождеству $x^{2}=0$, с неразрешимой эквачиональной теорией.

ДокАЗАТЕЛЬСтво. Введем ряд обозначений. Пусть $y^{0}-$ пустой символ,

$$
x y^{n}=\left(\cdots(x \underbrace{y) \cdots) y}_{n \text { раз }}, \quad E(x, y)=\left(\left(\left(x y^{3}\right)\left(x y^{4}\right)\right)\left(\left(x y^{4}\right)\left(x y^{5}\right)\right)\right),\right.
$$

$P$ - рекурсивно перечислимое нерекурсивное множество натуральных чисел. Обозначим через М двухленточную машину Минского, вычисляющую частичную характеристическую функцию множества $P$. Пусть $m+1$ - число внутренних состояний машины M 
и $A, B, C, D, Q_{0}, Q_{1}, \ldots, Q_{m}$ - следующие слова:

$$
\begin{gathered}
A(x, y)=\left(E(x, y) y^{2}\right) E(x, y), \quad B(x, y)=\left(E(x, y) y^{3}\right) E(x, y), \\
C(x, y)=\left(E(x, y) y^{4}\right) E(x, y), \quad D(x, y)=\left(E(x, y) y^{5}\right) E(x, y), \\
Q_{i}(x, y)=\left(E(x, y) y^{i+6}\right) E(x, y) .
\end{gathered}
$$

Пусть $W=\left\{A, B, C, D, Q_{i} \mid i \in\{0,1, \ldots, m\}\right\}$. Пусть $\mathfrak{J}$ - многообразие колец, заданное тождествами $2 x=0, x^{2}=0 ; F_{n} \mathfrak{J}$ - кольцо ранга $n$, свободное в многообразии $\mathfrak{J}$, имеющее множество свободных образующих $\left\{e_{1}, e_{2}, e_{3}, \ldots\right\}$.

Произвольной команде $q_{i} \delta_{1} \delta_{2} \rightarrow q_{j} \varepsilon_{1} \varepsilon_{2}$ машины М поставим в соответствие некоторое кольцевое тождество:

$Q_{i}(x, y) C(x, y) D(x, y)=Q_{j}(x, y) A^{\varepsilon_{1}}(x, y) B^{\varepsilon_{2}}(x, y) C(x, y) D(x, y)$, если $\delta_{1}=1, \delta_{2}=1$, $Q_{i}(x, y) A(x, y) D(x, y)=Q_{j}(x, y) A^{\varepsilon_{1}+1}(x, y) B^{\varepsilon_{2}}(x, y) D(x, y), \quad$ если $\delta_{1}=1, \delta_{2}=0$, $Q_{i}(x, y) B(x, y) C(x, y)=Q_{j}(x, y) A^{\varepsilon_{1}}(x, y) B^{\varepsilon_{2}+1}(x, y) C(x, y), \quad$ если $\delta_{1}=0, \delta_{2}=1$, $Q_{i}(x, y) A(x, y) B(x, y)=Q_{j}(x, y) A^{\varepsilon_{1}+1}(x, y) B^{\varepsilon_{2}+1}(x, y), \quad$ если $\delta_{1}=0, \delta_{2}=0$.

Пусть $\mathfrak{B}$ - многообразие колец, заданное тождествами

$$
\begin{array}{ll}
z A(x, y) B(x, y)=z B(x, y) A(x, y), & z A(x, y) D(x, y)=z D(x, y) A(x, y) \\
z C(x, y) B(x, y)=z B(x, y) C(x, y), & z C(x, y) D(x, y)=z D(x, y) C(x, y)
\end{array}
$$

$\mathfrak{M}$ - многообразие колец, заданное тождествами, кодирующими команды машины М, $\mathfrak{X}=\mathfrak{B} \cap \mathfrak{M} \cap \mathfrak{J}$.

ЛЕмма 1. Если в многообразии $\mathfrak{X}$ выполняется тождество

$$
Q_{1}(x, y) A^{2^{n}}(x, y) C(x, y) D(x, y)=Q_{j}(x, y) A(x, y) C(x, y) D(x, y),
$$

mo $n \in P$.

ДокАЗАТЕльство. Обозначим через $F_{k} \mathfrak{X}$ кольцо ранга $k$, свободное в многообразии $\mathfrak{X}$. Для удобства образующие кольца $F_{k} \mathfrak{X}$ будем обозначать теми же буквами, что и образующие кольца $F \mathfrak{J}$, а тождество $(1)-\mathfrak{F}(x, y)=0$. Так как тождество (1) выполняется в многообразии $\mathfrak{X}$, оно вьполняется и в кольце $F_{\omega} \mathfrak{X}$. Отсюда вытекает, что в кольце $F_{\omega} \mathfrak{X}$ истинно соотношение $\mathfrak{F}\left(e_{1}, e_{2}\right)=0$. Обозначим через $S$ множество многочленов $f(\vec{e})$ таких, что $f(\vec{e})=0$ - соотношение в кольце $F_{\omega} \mathfrak{X}$, полученное из одного из тождеств, задаюших многообразие $\mathfrak{X}$, подстановкой вместо переменных элементов кольца $F_{\omega} \mathfrak{X}$. Пусть $I$ - идеал кольца $F_{\omega} \mathfrak{X}$, порожденньй множеством $S$. Очевидно, что $F_{\omega} \mathfrak{X}=\mathfrak{F}\left(e_{1}, e_{2}\right)=0$ тогда и только тогда, когда $F_{\omega} \mathfrak{J}=\mathfrak{F}\left(e_{1}, e_{2}\right) \in I$. Из соотношения $F_{\omega} \mathfrak{J} \models \mathfrak{F}\left(e_{1}, e_{2}\right) \in I$ вытекает, что в кольце $F_{\omega} \mathfrak{J}$ вьполняется равенство следующего вида:

$$
\mathfrak{F}\left(e_{1}, e_{2}\right)=\sum_{i, j} u_{i_{1}} h_{j} u_{i_{2}},
$$

где $h_{j} \in S, u_{i_{1}} h_{j} u_{i_{2}}$ - некоторое произведение многочленов типа $u_{i_{1}}{ }^{(1)} \cdots u_{i_{1}}{ }^{(k)} h_{j} u_{i_{2}}{ }^{(1)}$ $\cdots u_{i_{2}}{ }^{(l)}$ с произвольньм порядком расположения скобок. В силу дистрибутивности можно считать, что все многочлены, входящие в $u_{i_{1}} h_{j} u_{i_{2}}$, кроме $h_{j}$, являются одночленами.

Пусть $f_{1}=\sum_{i, j} v_{i_{1}} h_{j} v_{i_{2}}$ и каждьй одночлен многочлена $f_{1}$ для некоторых $w_{i} \in W$ имеет вид $w_{1}\left(e_{1}, e_{2}\right) \cdots w_{k}\left(e_{1}, e_{2}\right)$, причем в многочлене $f_{1}$ для некоторого $j \in\{0,1$, $\ldots, m\} w_{1}=Q_{j}$ и для любых $l_{1}, l_{2} w_{l_{1}} \neq Q_{l_{2}}$ при условии $l_{1} \geqslant 2, f_{2}$ - многочлен, ни один одночлен которого ни для каких $j \in\{0,1, \ldots, m\}$ и $w_{i} \in W \backslash\left\{Q_{i} \mid i \in\{0,1, \ldots, m\}\right\}$ не равен $Q_{j}\left(e_{1}, e_{2}\right) w_{1}\left(e_{1}, e_{2}\right) w_{2}\left(e_{1}, e_{2}\right) \cdots w_{k}\left(e_{1}, e_{2}\right)$. 
Лемма 2. Существуют $f_{1} u f_{2}$ такие, ито $f_{1}+f_{2}=\sum_{i, j} u_{i_{1}} h_{j} u_{i_{2}}$.

ДоКАЗАТЕЛЬСТВо. Воспользуемся индукцией по числу одночленов в многочлене $\sum_{i, j} u_{i_{1}} h_{j} u_{i_{2}}$. Допустим, что число $d$ одночленов в сумме $\sum_{i, j} u_{i_{1}} h_{j} u_{i_{2}}$ равно 0 . Тогда утверждение очевидно. Пусть утверждение справедливо для некоторого числа одночленов $d$. Покажем, что оно верно и для $d+1$. Пусть число одночленов в сумме $\sum_{i, j} u_{i_{1}} h_{j} u_{i_{2}}$ не больше $d+1$. Тогда

$$
\sum_{i, j} u_{i_{1}} h_{j} u_{i_{2}}=\sum_{i>1, j} u_{i_{1}} h_{j} u_{i_{2}}+u_{1_{1}} h_{1} u_{1_{2}} .
$$

Так как число одночленов в сумме $\sum_{i>1, j} u_{i_{1}} h_{j} u_{i_{2}}$ не превосходит $d$, по предположению индукции имеем $f_{1}+f_{2}=\sum_{i>1, j} u_{i_{1}} h_{j} u_{i_{2}}$. Рассмотрим многочлен $u_{1_{1}} h_{1} u_{1_{2}}$. В зависимости от того, получен ли многочлен $h_{1}$ из тождества многообразия $\mathfrak{M}$ или из тождества многообразия $\mathfrak{B}$, следует рассмотреть два случая.

Случай многообразия $\mathfrak{M}$. Так как многочлен $h_{1}$ получен из тождества многообразия $\mathfrak{M}$, он имеет вид $w_{i_{1}}(x, y) w_{i_{2}}(x, y) \cdots w_{i_{s}}(x, y)-w_{j_{1}}(x, y) w_{j_{2}}(x, y) \cdots w_{j_{t}}(x, y)$. Без ограничения общности можно считать, что в многочлен $\sum_{i, j} u_{i_{1}} h_{j} u_{i_{2}}$ входят только образуюшие $e_{1}$ и $e_{2}$. Следовательно, $x=\alpha e_{1}+\beta e_{2}+\bar{x}\left(e_{1}, e_{2}\right), y=\gamma e_{1}+\delta e_{2}+\bar{y}\left(e_{1}, e_{2}\right)$, где $\alpha, \beta, \gamma, \delta \in\{0,1\}, \bar{x}\left(e_{1}, e_{2}\right), \bar{y}\left(e_{1}, e_{2}\right)$ - многочлены от $e_{1}, e_{2}$, нижняя степень которых не меньше 2. Тогда

$$
\begin{aligned}
& w_{i_{1}}(x, y) w_{i_{2}}(x, y) \cdots w_{i_{s}}(x, y)-w_{j_{1}}(x, y) w_{j_{2}}(x, y) \cdots w_{j_{t}}(x, y) \\
&= w_{i_{1}}\left(\alpha e_{1}+\beta e_{2}+\bar{x}\left(e_{1}, e_{2}\right), \gamma e_{1}+\delta e_{2}+\bar{y}\left(e_{1}, e_{2}\right)\right) \\
& w_{i_{2}}\left(\alpha e_{1}+\beta e_{2}+\bar{x}\left(e_{1}, e_{2}\right), \gamma e_{1}+\delta e_{2}+\bar{y}\left(e_{1}, e_{2}\right)\right) \cdots \\
& w_{i_{s}}\left(\alpha e_{1}+\beta e_{2}+\bar{x}\left(e_{1}, e_{2}\right), \gamma e_{1}+\delta e_{2}+\bar{y}\left(e_{1}, e_{2}\right)\right) \\
&- w_{j_{1}}\left(\alpha e_{1}+\beta e_{2}+\bar{x}\left(e_{1}, e_{2}\right), \gamma e_{1}+\delta e_{2}+\bar{y}\left(e_{1}, e_{2}\right)\right) \\
& w_{j_{2}}\left(\alpha e_{1}+\beta e_{2}+\bar{x}\left(e_{1}, e_{2}\right), \gamma e_{1}+\delta e_{2}+\bar{y}\left(e_{1}, e_{2}\right)\right) \cdots \\
& w_{j_{t}}\left(\alpha e_{1}+\beta e_{2}+\bar{x}\left(e_{1}, e_{2}\right), \gamma e_{1}+\delta e_{2}+\bar{y}\left(e_{1}, e_{2}\right)\right) \\
&= w_{i_{1}}\left(\alpha e_{1}+\beta e_{2}, \gamma e_{1}+\delta e_{2}\right) w_{i_{2}}\left(\alpha e_{1}+\beta e_{2}, \gamma e_{1}+\delta e_{2}\right) \cdots w_{i_{s}}\left(\alpha e_{1}+\beta e_{2}, \gamma e_{1}+\delta e_{2}\right) \\
&- w_{j_{1}}\left(\alpha e_{1}+\beta e_{2}, \gamma e_{1}+\delta e_{2}\right) w_{j_{2}}\left(\alpha e_{1}+\beta e_{2}, \gamma e_{1}+\delta e_{2}\right) \cdots w_{j_{t}}\left(\alpha e_{1}+\beta e_{2}, \gamma e_{1}+\delta e_{2}\right) \\
&+ U\left(e_{1}, e_{2}\right),
\end{aligned}
$$

где $U\left(e_{1}, e_{2}\right)$ - многочлен, в каждьй одночлен которого в качестве подслова входит одночлен многочлена $\bar{x}\left(e_{1}, e_{2}\right)$ или $\bar{y}\left(e_{1}, e_{2}\right)$. Покажем, что каждый одночлен многочлена $U\left(e_{1}, e_{2}\right)$ является одночленом многочлена $f_{2}$. Допустим противное. Тогда в кольце $F_{\omega} \mathfrak{J}$ должно выполняться равенство следующего вида:

$$
w_{l_{1}}\left(e_{1}, e_{2}\right) \cdots w_{l_{r_{1}}}\left(e_{1}, e_{2}\right)=w_{p_{1}}\left(\eta_{1_{p_{1}}}, \eta_{2_{p_{1}}}\right) \cdots w_{p_{r_{2}}}\left(\eta_{1_{p_{r_{2}}}}, \eta_{2_{p_{r_{2}}}}\right),
$$

где $\eta \in\left\{e_{1}, e_{2}, z \mid z \neq e_{1}, z \neq e_{2}, z\right.$-слово от $\left.e_{1}, e_{2}\right\}$, причем существует $\eta$ отличное от $e_{1}$ и $e_{2}$.

\section{Лемма 3. Равенство (2) ложсно.}

ДокАЗАТЕЛЬСтво. Докажем индукцией по числу $r_{2}$. Предположим, что $r_{2}=1$. Тогда равенство (2) имеет вид

$$
w_{l_{1}}\left(e_{1}, e_{2}\right) \cdots w_{l_{r_{1}}}\left(e_{1}, e_{2}\right)=w_{p_{1}}\left(\eta_{1_{p_{1}}}, \eta_{2_{p_{1}}}\right) .
$$




\section{Лемма 4. Равенство (3) ложно.}

ДоКАЗАТЕЛЬСТВо. Применим индукцию по числу $r_{1}$. Предположим, что $r_{1}=1$. Тогда равенство (3) имеет вид $w_{l_{1}}\left(e_{1}, e_{2}\right)=w_{p_{1}}\left(\eta_{1_{p_{1}}}, \eta_{2_{p_{1}}}\right)$. Поэтому

$$
\begin{aligned}
& \left(E\left(e_{1}, e_{2}\right) e_{2}^{s}\right) E\left(e_{1}, e_{2}\right) \\
& \quad=\left(\left(( \cdots ) \left(( ( ( ( \eta _ { 1 } \eta _ { 2 } ) \eta _ { 3 } ) \eta _ { 4 } ) ( ( ( ( \eta _ { 5 } \eta _ { 6 } ) \eta _ { 7 } ) \eta _ { 8 } ) \eta _ { 9 } ) ) \left(\left(\left(\left(\left(\eta_{10} \eta_{11}\right) \eta_{12}\right) \eta_{13}\right) \eta_{14}\right)\right.\right.\right.\right. \\
& \left.\left.\left.\left.\left.\quad\left(\left(\left(\left(\left(\eta_{15} \eta_{16}\right) \eta_{17}\right) \eta_{18}\right) \eta_{19}\right) \eta_{20}\right)\right)\right) \eta_{21}\right) \cdots\right) \eta_{t+20}\right)\left(\left(\left(\left(\left(\eta_{t+21} \eta_{t+22}\right) \eta_{t+23}\right) \eta_{t+24}\right)\right.\right. \\
& \left.\quad\left(\left(\left(\left(\eta_{t+25} \eta_{t+26}\right) \eta_{t+27}\right) \eta_{t+28}\right) \eta_{t+29}\right)\right)\left(\left(\left(\left(\left(\eta_{t+30} \eta_{t+31}\right) \eta_{t+32}\right) \eta_{t+33}\right) \eta_{t+34}\right)\right. \\
& \left.\left.\left.\quad\left(\left(\left(\left(\left(\eta_{t+35} \eta_{t+36}\right) \eta_{t+37}\right) \eta_{t+38}\right) \eta_{t+39}\right) \eta_{t+40}\right)\right)\right)\right) .
\end{aligned}
$$

В силу свободы кольца $F_{\omega} \mathfrak{J}$ это равенство равносильно одной из следующих двух систем равенств:

$$
\begin{aligned}
& E\left(e_{1}, e_{2}\right) e_{2}^{s}=\left(( \cdots ) \left(\left(\left(\left(\left(\eta_{1} \eta_{2}\right) \eta_{3}\right) \eta_{4}\right)\left(\left(\left(\left(\eta_{5} \eta_{6}\right) \eta_{7}\right) \eta_{8}\right) \eta_{9}\right)\right)\right.\right. \\
& \left.\left.\left.\left.\quad\left(\left(\left(\left(\left(\eta_{10} \eta_{11}\right) \eta_{12}\right) \eta_{13}\right) \eta_{14}\right)\left(\left(\left(\left(\left(\eta_{15} \eta_{16}\right) \eta_{17}\right) \eta_{18}\right) \eta_{19}\right) \eta_{20}\right)\right)\right) \eta_{21}\right) \cdots\right) \eta_{t+20}\right) \\
& E\left(e_{1}, e_{2}\right)=\left(\left(\left(\left(\left(\eta_{t+21} \eta_{t+22}\right) \eta_{t+23}\right) \eta_{t+24}\right)\left(\left(\left(\left(\eta_{t+25} \eta_{t+26}\right) \eta_{t+27}\right) \eta_{t+28}\right) \eta_{t+29}\right)\right)\right. \\
& \left.\quad\left(\left(\left(\left(\left(\eta_{t+30} \eta_{t+31}\right) \eta_{t+32}\right) \eta_{t+33}\right) \eta_{t+34}\right)\left(\left(\left(\left(\left(\eta_{t+35} \eta_{t+36}\right) \eta_{t+37}\right) \eta_{t+38}\right) \eta_{t+39}\right) \eta_{t+40}\right)\right)\right) \\
& E\left(e_{1}, e_{2}\right) e_{2}^{s}=\left(\left(\left(\left(\left(\eta_{t+21} \eta_{t+22}\right) \eta_{t+23}\right) \eta_{t+24}\right)\left(\left(\left(\left(\eta_{t+25} \eta_{t+26}\right) \eta_{t+27}\right) \eta_{t+28}\right) \eta_{t+29}\right)\right)\right. \\
& \left.\quad\left(\left(\left(\left(\left(\eta_{t+30} \eta_{t+31}\right) \eta_{t+32}\right) \eta_{t+33}\right) \eta_{t+34}\right)\left(\left(\left(\left(\left(\eta_{t+35} \eta_{t+36}\right) \eta_{t+37}\right) \eta_{t+38}\right) \eta_{t+39}\right) \eta_{t+40}\right)\right)\right) \\
& E\left(e_{1}, e_{2}\right)=\left(\left(\cdots \left(( ( ( ( \eta _ { 1 } \eta _ { 2 } ) \eta _ { 3 } ) \eta _ { 4 } ) ( ( ( ( \eta _ { 5 } \eta _ { 6 } ) \eta _ { 7 } ) \eta _ { 8 } ) \eta _ { 9 } ) ) \left(\left(\left(\left(\left(\eta_{10} \eta_{11}\right) \eta_{12}\right) \eta_{13}\right) \eta_{14}\right)\right.\right.\right.\right. \\
& \left.\left.\left.\left.\left.\quad\left(\left(\left(\left(\left(\eta_{15} \eta_{16}\right) \eta_{17}\right) \eta_{18}\right) \eta_{19}\right) \eta_{20}\right)\right)\right) \eta_{21}\right) \cdots\right) \eta_{t+20}\right)
\end{aligned}
$$

Рассмотрим систему равенств (4).

Лемма 5. Если выполняется первое равенство системы (4), то для любого $i \in\{1, \ldots, t+20\} \quad \eta_{i} \in\left\{e_{1}, e_{2}\right\}$.

ДокАЗАТЕЛЬСтво. Доказательство проведем индукцией по числу $t$. Заметим, что по построению многочлена $w_{p_{1}}\left(\eta_{1_{p_{1}}}, \eta_{2_{p_{1}}}\right)$ число $t$ больше 1 . Предположим, что $t=2$. Тогда

$$
\begin{aligned}
E\left(e_{1}, e_{2}\right) e_{2}^{s}= & \left(\left(\left(( ( ( ( \eta _ { 1 } \eta _ { 2 } ) \eta _ { 3 } ) \eta _ { 4 } ) ( ( ( ( \eta _ { 5 } \eta _ { 6 } ) \eta _ { 7 } ) \eta _ { 8 } ) \eta _ { 9 } ) ) \left(\left(\left(\left(\left(\eta_{10} \eta_{11}\right) \eta_{12}\right) \eta_{13}\right) \eta_{14}\right)\right.\right.\right.\right. \\
& \left.\left.\left.\left.\left(\left(\left(\left(\left(\eta_{15} \eta_{16}\right) \eta_{17}\right) \eta_{18}\right) \eta_{19}\right) \eta_{20}\right)\right)\right) \eta_{21}\right) \eta_{22}\right) .
\end{aligned}
$$

Значит, в силу свободы кольца $F_{\omega} \mathfrak{J}$ вьполняется одна из следующих систем равенств:

$$
\begin{gathered}
E\left(e_{1}, e_{2}\right) e_{2}^{s-1}=\left(\left(\left(\left(\left(\left(\eta_{1} \eta_{2}\right) \eta_{3}\right) \eta_{4}\right)\left(\left(\left(\left(\eta_{5} \eta_{6}\right) \eta_{7}\right) \eta_{8}\right) \eta_{9}\right)\right)\right.\right. \\
\left.\left.\left.\quad\left(\left(\left(\left(\eta_{10} \eta_{11}\right) \eta_{12}\right) \eta_{13}\right) \eta_{14}\right)\left(\left(\left(\left(\left(\eta_{15} \eta_{16}\right) \eta_{17}\right) \eta_{18}\right) \eta_{19}\right) \eta_{20}\right)\right)\right) \eta_{21}\right), \\
e_{2}=\eta_{22} \\
E\left(e_{1}, e_{2}\right) e_{2}^{s-1}=\eta_{22} \\
\left(\left(\left(\left(\left(\left(\eta_{1} \eta_{2}\right) \eta_{3}\right) \eta_{4}\right)\left(\left(\left(\left(\eta_{5} \eta_{6}\right) \eta_{7}\right) \eta_{8}\right) \eta_{9}\right)\right)\left(\left(\left(\left(\eta_{10} \eta_{11}\right) \eta_{12}\right) \eta_{13}\right) \eta_{14}\right)\right.\right. \\
\left.\left.\left.\quad\left(\left(\left(\left(\left(\eta_{15} \eta_{16}\right) \eta_{17}\right) \eta_{18}\right) \eta_{19}\right) \eta_{20}\right)\right)\right) \eta_{21}\right)=e_{2}
\end{gathered}
$$

Заметим, что длина слова $e_{2}$ равна 1 , а длина слова

$$
\begin{aligned}
& \quad\left(( ( ( ( \eta _ { 1 } \eta _ { 2 } ) \eta _ { 3 } ) \eta _ { 4 } ) ( ( ( ( \eta _ { 5 } \eta _ { 6 } ) \eta _ { 7 } ) \eta _ { 8 } ) \eta _ { 9 } ) ) \left(\left(\left(\left(\left(\eta_{10} \eta_{11}\right) \eta_{12}\right) \eta_{13}\right) \eta_{14}\right)\right.\right. \\
& \left.\left.\left.\quad\left(\left(\left(\left(\left(\eta_{15} \eta_{16}\right) \eta_{17}\right) \eta_{18}\right) \eta_{19}\right) \eta_{20}\right)\right)\right) \eta_{21}\right)
\end{aligned}
$$


больше 21. Следовательно, второе равенство системы (7) ложно. Рассмотрим систему (6). Первое равенство системы (6) равносильно следующей системе:

$$
\begin{gathered}
E\left(e_{1}, e_{2}\right) e_{2}^{s-2}=\left(\left(\left(\left(\left(\eta_{1} \eta_{2}\right) \eta_{3}\right) \eta_{4}\right)\left(\left(\left(\left(\eta_{5} \eta_{6}\right) \eta_{7}\right) \eta_{8}\right) \eta_{9}\right)\right)\right. \\
\left.\left.\left(\left(\left(\left(\eta_{10} \eta_{11}\right) \eta_{12}\right) \eta_{13}\right) \eta_{14}\right)\left(\left(\left(\left(\left(\eta_{15} \eta_{16}\right) \eta_{17}\right) \eta_{18}\right) \eta_{19}\right) \eta_{20}\right)\right)\right) \\
e_{2}=\eta_{21}
\end{gathered}
$$

или

$$
\begin{gathered}
E\left(e_{1}, e_{2}\right) e_{2}^{s-2}=\eta_{21} \\
\left(\left(\left(\left(\eta_{1} \eta_{2}\right) \eta_{3}\right) \eta_{4}\right)\left(\left(\left(\left(\eta_{5} \eta_{6}\right) \eta_{7}\right) \eta_{8}\right) \eta_{9}\right)\right)\left(\left(\left(\left(\left(\eta_{10} \eta_{11}\right) \eta_{12}\right) \eta_{13}\right) \eta_{14}\right)\right. \\
\left.\left.\left(\left(\left(\left(\left(\eta_{15} \eta_{16}\right) \eta_{17}\right) \eta_{18}\right) \eta_{19}\right) \eta_{20}\right)\right)\right)=e_{2}
\end{gathered}
$$

Легко понять, что система (9) ложна. Следовательно, должна выполняться система (8). Предположим, что $s-2>0$. Тогда из первого равенства системы (8) получаем

$$
\begin{gathered}
E\left(e_{1}, e_{2}\right) e_{2}^{s-3}=\left(\left(\left(\left(\eta_{1} \eta_{2}\right) \eta_{3}\right) \eta_{4}\right)\left(\left(\left(\left(\eta_{5} \eta_{6}\right) \eta_{7}\right) \eta_{8}\right) \eta_{9}\right)\right) \\
e_{2}=\left(\left(\left(\left(\left(\left(\eta_{10} \eta_{11}\right) \eta_{12}\right) \eta_{13}\right) \eta_{14}\right)\left(\left(\left(\left(\left(\eta_{15} \eta_{16}\right) \eta_{17}\right) \eta_{18}\right) \eta_{19}\right) \eta_{20}\right)\right)\right) \\
E\left(e_{1}, e_{2}\right) e_{2}^{s-3}=\left(\left(\left(\left(\left(\eta_{10} \eta_{11}\right) \eta_{12}\right) \eta_{13}\right) \eta_{14}\right)\left(\left(\left(\left(\left(\eta_{15} \eta_{16}\right) \eta_{17}\right) \eta_{18}\right) \eta_{19}\right) \eta_{20}\right)\right) \\
e_{2}=\left(\left(\left(\left(\eta_{1} \eta_{2}\right) \eta_{3}\right) \eta_{4}\right)\left(\left(\left(\left(\eta_{5} \eta_{6}\right) \eta_{7}\right) \eta_{8}\right) \eta_{9}\right)\right)
\end{gathered}
$$

Длина слова $\left(\left(\left(\left(\eta_{1} \eta_{2}\right) \eta_{3}\right) \eta_{4}\right)\left(\left(\left(\left(\eta_{5} \eta_{6}\right) \eta_{7}\right) \eta_{8}\right) \eta_{9}\right)\right)$ больше 9 , а длина слова $\left(\left(\left(\left(\eta_{10} \eta_{11}\right) \eta_{12}\right)\right.\right.$ $\left.\left.\left.\left.\eta_{13}\right) \eta_{14}\right)\left(\left(\left(\left(\eta_{15} \eta_{16}\right) \eta_{17}\right) \eta_{18}\right) \eta_{19}\right) \eta_{20}\right)\right)$ больше 11 . Так как длина слова $e_{2}$ равна 1 , в силу свободы кольца $F_{\omega} \mathfrak{J}$ системы равенств $(10)$ и $(11)$ ложны. Допустим теперь, что $s-2=0$. Тогда первое равенство системы (8) имеет следующий вид:

$$
\begin{aligned}
E\left(e_{1}, e_{2}\right)= & \left(( ( ( ( \eta _ { 1 } \eta _ { 2 } ) \eta _ { 3 } ) \eta _ { 4 } ) ( ( ( ( \eta _ { 5 } \eta _ { 6 } ) \eta _ { 7 } ) \eta _ { 8 } ) \eta _ { 9 } ) ) \left(\left(\left(\left(\left(\eta_{10} \eta_{11}\right) \eta_{12}\right) \eta_{13}\right) \eta_{14}\right)\right.\right. \\
& \left.\left.\left(\left(\left(\left(\left(\eta_{15} \eta_{16}\right) \eta_{17}\right) \eta_{18}\right) \eta_{19}\right) \eta_{20}\right)\right)\right) .
\end{aligned}
$$

Отсюда вытекает одна из следующих систем:

$$
\begin{aligned}
& \left(\left(\left(\left(\left(e_{1} e_{2}\right) e_{2}\right) e_{2}\right) e_{2}\right)\left(\left(\left(\left(\left(e_{1} e_{2}\right) e_{2}\right) e_{2}\right) e_{2}\right) e_{2}\right)\right)=\left(\left(\left(\left(\left(\eta_{1} \eta_{2}\right) \eta_{3}\right) \eta_{4}\right)\left(\left(\left(\left(\eta_{5} \eta_{6}\right) \eta_{7}\right) \eta_{8}\right) \eta_{9}\right)\right)\right) \\
& \left(\left(\left(\left(e_{1} e_{2}\right) e_{2}\right) e_{2}\right)\left(\left(\left(\left(e_{1} e_{2}\right) e_{2}\right) e_{2}\right) e_{2}\right)\right) \\
& \quad=\left(\left(\left(\left(\left(\eta_{10} \eta_{11}\right) \eta_{12}\right) \eta_{13}\right) \eta_{14}\right)\left(\left(\left(\left(\left(\eta_{15} \eta_{16}\right) \eta_{17}\right) \eta_{18}\right) \eta_{19}\right) \eta_{20}\right)\right) \\
& \left.\quad\left(\left(\left(\left(e_{1} e_{2}\right) e_{2}\right) e_{2}\right)\left(\left(\left(\left(e_{1} e_{2}\right) e_{2}\right) e_{2}\right) e_{2}\right)\right)=\left(\left(\left(\left(\eta_{1} \eta_{2}\right) \eta_{3}\right) \eta_{4}\right)\left(\left(\left(\left(\eta_{5} \eta_{6}\right) \eta_{7}\right) \eta_{8}\right) \eta_{9}\right)\right)\right) \\
& \quad\left(\left(\left(\left(\left(e_{1} e_{2}\right) e_{2}\right) e_{2}\right) e_{2}\right)\left(\left(\left(\left(\left(e_{1} e_{2}\right) e_{2}\right) e_{2}\right) e_{2}\right) e_{2}\right)\right) \\
& \quad=\left(\left(\left(\left(\left(\eta_{10} \eta_{11}\right) \eta_{12}\right) \eta_{13}\right) \eta_{14}\right)\left(\left(\left(\left(\left(\eta_{15} \eta_{16}\right) \eta_{17}\right) \eta_{18}\right) \eta_{19}\right) \eta_{20}\right)\right)
\end{aligned}
$$

Рассмотрим систему равенств (12). Из второго равенства системы (12) получаем

$$
\begin{gathered}
\left(\left(e_{1} e_{2}\right) e_{2}\right) e_{2}=\left(\left(\left(\eta_{10} \eta_{11}\right) \eta_{12}\right) \eta_{13}\right) \eta_{14}, \\
\left(\left(\left(\left(\left(e_{1} e_{2}\right) e_{2}\right) e_{2}\right) e_{2}\right)\right)=\left(\left(\left(\left(\eta_{15} \eta_{16}\right) \eta_{17}\right) \eta_{18}\right) \eta_{19}\right) \eta_{20} \\
\left(\left(e_{1} e_{2}\right) e_{2}\right) e_{2}=\left(\left(\left(\left(\eta_{15} \eta_{16}\right) \eta_{17}\right) \eta_{18}\right) \eta_{19}\right) \eta_{20}, \\
\left(\left(\left(\left(\left(e_{1} e_{2}\right) e_{2}\right) e_{2}\right) e_{2}\right)\right)=\left(\left(\left(\eta_{10} \eta_{11}\right) \eta_{12}\right) \eta_{13}\right) \eta_{14} .
\end{gathered}
$$

Легко понять, что системы (14) и (15) ложны. Рассмотрим систему (13).

ЛЕмма 6. Если выполняется система (13), то для любого $i \in\{1, \ldots, t+20\}$ $\eta_{i} \in\left\{e_{1}, e_{2}\right\}$. 
ДокАЗАТЕЛЬСтво. Из первого равенства системы (13) получаем

$$
\begin{aligned}
& \left(\left(e_{1} e_{2}\right) e_{2}\right) e_{2}=\left(\left(\left(\eta_{5} \eta_{6}\right) \eta_{7}\right) \eta_{8}\right) \eta_{9}, \quad\left(\left(\left(e_{1} e_{2}\right) e_{2}\right) e_{2}\right) e_{2}=\left(\left(\eta_{1} \eta_{2}\right) \eta_{3}\right) \eta_{4} \\
& \left(\left(e_{1} e_{2}\right) e_{2}\right) e_{2}=\left(\left(\eta_{1} \eta_{2}\right) \eta_{3}\right) \eta_{4}, \quad\left(\left(\left(e_{1} e_{2}\right) e_{2}\right) e_{2}\right) e_{2}=\left(\left(\left(\eta_{5} \eta_{6}\right) \eta_{7}\right) \eta_{8}\right) \eta_{9}
\end{aligned}
$$

Так как длина слова $\left(\left(e_{1} e_{2}\right) e_{2}\right) e_{2}$ равна 4 , а длина слова $\left(\left(\left(\eta_{5} \eta_{6}\right) \eta_{7}\right) \eta_{8}\right) \eta_{9}$ больше 5 , paвенство $\left(\left(e_{1} e_{2}\right) e_{2}\right) e_{2}=\left(\left(\left(\eta_{5} \eta_{6}\right) \eta_{7}\right) \eta_{8}\right) \eta_{9}$ ложно. Следовательно, ложна и система (16).

Рассмотрим систему (17). Из первого равенства системы (17) получаем

$$
\begin{aligned}
& \left(e_{1} e_{2}\right) e_{2}=\eta_{4}, \quad e_{2}=\left(\eta_{1} \eta_{2}\right) \eta_{3} \\
& \left(e_{1} e_{2}\right) e_{2}=\left(\eta_{1} \eta_{2}\right) \eta_{3}, \quad e_{2}=\eta_{4}
\end{aligned}
$$

Легко понять, что $e_{2} \neq\left(\eta_{1} \eta_{2}\right) \eta_{3}$. Поэтому система (18) неверна. Из системы (19) получаем

$$
\begin{aligned}
& e_{1} e_{2}=\eta_{3}, \quad e_{2}=\eta_{1} \eta_{2} ; \\
& e_{1} e_{2}=\eta_{1} \eta_{2}, \quad e_{2}=\eta_{3} .
\end{aligned}
$$

Так как $e_{2} \neq \eta_{1} \eta_{2}$, система (20) ложна. Следовательно, имеет место система (21), из которой получаем $\eta_{1}=e_{1}, \eta_{2}=e_{2}$ или $\eta_{1}=e_{2}, \eta_{2}=e_{1}$. Таким образом, если вьполняется первое равенство системы (17), то для любого $i \in\{1, \ldots, 4\} \eta_{i} \in\left\{e_{1}, e_{2}\right\}$. Из второго равенства системы (17) вытекают равенства

$$
\begin{gathered}
\left(\left(e_{1} e_{2}\right) e_{2}\right) e_{2}=\eta_{9}, \quad e_{2}=\left(\left(\eta_{5} \eta_{6}\right) \eta_{7}\right) \eta_{8} \\
\left(\left(e_{1} e_{2}\right) e_{2}\right) e_{2}=\left(\left(\left(\eta_{5} \eta_{6}\right) \eta_{7}\right) \eta_{8}\right), \quad e_{2}=\eta_{9}
\end{gathered}
$$

Так как $e_{2} \neq\left(\left(\eta_{5} \eta_{6}\right) \eta_{7}\right) \eta_{8}$, система $(22)$ ложна. Поэтому

$$
\begin{aligned}
& \left(e_{1} e_{2}\right) e_{2}=\eta_{8}, \quad e_{2}=\left(\eta_{5} \eta_{6}\right) \eta_{7} \\
& \left(e_{1} e_{2}\right) e_{2}=\left(\eta_{5} \eta_{6}\right) \eta_{7}, \quad e_{2}=\eta_{8}
\end{aligned}
$$

Так как $e_{2} \neq\left(\eta_{5} \eta_{6}\right) \eta_{7}$, имеем (23). Из (23) вытекает, что для $i \in\{5,6,7\} \eta_{i} \in\left\{e_{1}, e_{2}\right\}$. Следовательно, для $i \in\{1, \ldots, 9\} \eta_{i} \in\left\{e_{1}, e_{2}\right\}$. Второе равенство системы (13) рассматривается совершенно аналогично. Лемма 6 доказана.

Таким образом, мы показали, что если вьполняется первое равенство системы (4) и $t=2$, то вьполняется система $(13)$ и $\eta_{21}, \eta_{22} \in\left\{e_{1}, e_{2}\right\}$. По лемме 6 получаем, что для $i \in\{1, \ldots, 22\} \eta_{i} \in\left\{e_{1}, e_{2}\right\}$. Следовательно, база индукции доказана.

Предположим, что лемма 5 доказана для всех $t \leqslant t_{0}$. Покажем, что лемма 5 справедлива и для $t=t_{0}+1$. Допустим, что первое равенство системы (4) справедливо при $t=t_{0}+1$. Тогда

$$
\begin{gathered}
E\left(e_{1}, e_{2}\right) e_{2}^{s-1}=\eta_{t_{0}+21} \\
\left(\left(\ldots \left(\left(\left(\left(\left(\eta_{1} \eta_{2}\right) \eta_{3}\right) \eta_{4}\right)\left(\left(\left(\left(\eta_{5} \eta_{6}\right) \eta_{7}\right) \eta_{8}\right) \eta_{9}\right)\right)\left(\left(\left(\left(\eta_{10} \eta_{11}\right) \eta_{12}\right) \eta_{13}\right) \eta_{14}\right)\right.\right.\right. \\
\left.\left.\left.\left.\left.\left(\left(\left(\left(\left(\eta_{15} \eta_{16}\right) \eta_{17}\right) \eta_{18}\right) \eta_{19}\right) \eta_{20}\right)\right)\right) \eta_{21}\right) \cdots\right) \eta_{t_{0}+20}\right)=e_{2} \\
E\left(e_{1}, e_{2}\right) e_{2}^{s-1}=\left(( \cdots ) \left(\left(\left(\left(\left(\eta_{1} \eta_{2}\right) \eta_{3}\right) \eta_{4}\right)\left(\left(\left(\left(\eta_{5} \eta_{6}\right) \eta_{7}\right) \eta_{8}\right) \eta_{9}\right)\right)\right.\right. \\
\left.\left.\left.\left.\left(\left(\left(\left(\left(\eta_{10} \eta_{11}\right) \eta_{12}\right) \eta_{13}\right) \eta_{14}\right)\left(\left(\left(\left(\left(\eta_{15} \eta_{16}\right) \eta_{17}\right) \eta_{18}\right) \eta_{19}\right) \eta_{20}\right)\right)\right) \eta_{21}\right) \cdots\right) \eta_{t_{0}+20}\right), \\
\eta_{t_{0}+21}=e_{2} .
\end{gathered}
$$

Легко понять, что система равенств (24) ложна. По предположению индукции из системы (25) вытекает утверждение леммы 5 для $t=t_{0}+1$. Лемма 5 доказана. 
ЛЕмма 7. Если выполняется второе равенство системы (4), то для любого $i \in\{t+21, \ldots, t+40\} \quad \eta_{i} \in\left\{e_{1}, e_{2}\right\}$.

ДокАЗАТЕЛЬСТво. Если вьполняется второе равенство системы (4), то должна выполняться одна из следующих двух систем равенств:

$$
\begin{aligned}
\left(\left(\left(e_{1} e_{2}\right) e_{2}\right) e_{2}\right)\left(\left(\left(\left(e_{1} e_{2}\right) e_{2}\right) e_{2}\right) e_{2}\right)= & \left(\left(\left(\left(\eta_{t+30} \eta_{t+31}\right) \eta_{t+32}\right) \eta_{t+33}\right) \eta_{t+34}\right) \\
& \left(\left(\left(\left(\left(\eta_{t+35} \eta_{t+36}\right) \eta_{t+37}\right) \eta_{t+38}\right) \eta_{t+39}\right) \eta_{t+40}\right), \\
\left(\left(\left(\left(e_{1} e_{2}\right) e_{2}\right) e_{2}\right) e_{2}\right)\left(\left(\left(\left(\left(e_{1} e_{2}\right) e_{2}\right) e_{2}\right) e_{2}\right) e_{2}\right)= & \left(\left(\left(\eta_{t+21} \eta_{t+22}\right) \eta_{t+23}\right) \eta_{t+24}\right) \\
& \left(\left(\left(\left(\eta_{t+25} \eta_{t+26}\right) \eta_{t+27}\right) \eta_{t+28}\right) \eta_{t+29}\right) ; \\
\left(\left(\left(e_{1} e_{2}\right) e_{2}\right) e_{2}\right)\left(\left(\left(\left(e_{1} e_{2}\right) e_{2}\right) e_{2}\right) e_{2}\right)= & \left(\left(\left(\eta_{t+21} \eta_{t+22}\right) \eta_{t+23}\right) \eta_{t+24}\right) \\
& \left(\left(\left(\left(\eta_{t+25} \eta_{t+26}\right) \eta_{t+27}\right) \eta_{t+28}\right) \eta_{t+29}\right) \\
\left(\left(\left(\left(e_{1} e_{2}\right) e_{2}\right) e_{2}\right) e_{2}\right)\left(\left(\left(\left(\left(e_{1} e_{2}\right) e_{2}\right) e_{2}\right) e_{2}\right) e_{2}\right)= & \left(\left(\left(\left(\eta_{t+30} \eta_{t+31}\right) \eta_{t+32}\right) \eta_{t+33}\right) \eta_{t+34}\right) \\
& \left(\left(\left(\left(\left(\eta_{t+35} \eta_{t+36}\right) \eta_{t+37}\right) \eta_{t+38}\right) \eta_{t+39}\right) \eta_{t+40}\right) .
\end{aligned}
$$

Легко понять, что система равенств (26) ложна. Рассмотрим систему (27). Из первого равенства системы (27) получаем

$$
\begin{gathered}
\left(\left(e_{1} e_{2}\right) e_{2}\right) e_{2}=\left(\left(\left(\eta_{t+25} \eta_{t+26}\right) \eta_{t+27}\right) \eta_{t+28}\right) \eta_{t+29} \\
\left(\left(\left(e_{1} e_{2}\right) e_{2}\right) e_{2}\right) e_{2}=\left(\left(\eta_{t+21} \eta_{t+22}\right) \eta_{t+23}\right) \eta_{t+24} \\
\left(\left(e_{1} e_{2}\right) e_{2}\right) e_{2}=\left(\left(\eta_{t+21} \eta_{t+22}\right) \eta_{t+23}\right) \eta_{t+24} \\
\left(\left(\left(e_{1} e_{2}\right) e_{2}\right) e_{2}\right) e_{2}=\left(\left(\left(\eta_{t+25} \eta_{t+26}\right) \eta_{t+27}\right) \eta_{t+28}\right) \eta_{t+29}
\end{gathered}
$$

Очевидно, что система (28) неверна. Из первого равенства системы (29), как нетрудно убедиться, получаем для $i \in\{t+21, \ldots, t+24\} \eta_{i} \in\left\{e_{1}, e_{2}\right\}$, а из второго равенства системы (29) для $i \in\{t+25, \ldots, t+29\} \eta_{i} \in\left\{e_{1}, e_{2}\right\}$. Второе равенство системы (27) рассматривается аналогично. Лемма 7 доказана.

Из лемм 5 и 7 получаем, что если выполняется система (4), то для любого $i \in\{1, \ldots$, $t+40\} \eta_{i} \in\left\{e_{1}, e_{2}\right\}$. Заметим, что второе равенство системы (5) в силу соотношения $t \geqslant 2$ ложно. Следовательно, равенство (3) ложно при $r_{1}=1$, так как в многочлене $w_{p_{1}}\left(\eta_{1 p_{1}}, \eta_{2 p_{1}}\right)$ для некоторого $i \eta_{i} \neq e_{1}$ и $\eta_{i} \neq e_{2}$.

Допустим, что лемма 4 справедлива для любого $r_{1} \leqslant r_{0}$. Покажем, что она верна и для $r_{1}=r_{0}+1$. При $r_{1}=r_{0}+1$ равенство (3) имеет вид

$$
\begin{aligned}
& w_{l_{1}}\left(e_{1}, e_{2}\right) \cdots w_{l_{r_{0}}}\left(e_{1}, e_{2}\right) w_{l_{r_{0}+1}}\left(e_{1}, e_{2}\right)=\left(\left(( \cdots ) \left(\left(\left(\left(\left(\eta_{1} \eta_{2}\right) \eta_{3}\right) \eta_{4}\right)\left(\left(\left(\left(\eta_{5} \eta_{6}\right) \eta_{7}\right) \eta_{8}\right) \eta_{9}\right)\right)\right.\right.\right. \\
& \left.\left.\left.\left.\quad\left(\left(\left(\left(\eta_{10} \eta_{11}\right) \eta_{12}\right) \eta_{13}\right) \eta_{14}\right)\left(\left(\left(\left(\left(\eta_{15} \eta_{16}\right) \eta_{17}\right) \eta_{18}\right) \eta_{19} \eta_{20}\right)\right)\right) \eta_{21}\right) \cdots\right) \eta_{t+20}\right) \\
& \quad\left(\left(\left(\left(\left(\eta_{t+21} \eta_{t+22}\right) \eta_{t+23}\right) \eta_{t+24}\right)\left(\left(\left(\left(\eta_{t+25} \eta_{t+26}\right) \eta_{t+27}\right) \eta_{t+28}\right) \eta_{t+29}\right)\right)\right. \\
& \left.\left.\quad\left(\left(\left(\left(\left(\eta_{t+30} \eta_{t+31}\right) \eta_{t+32}\right) \eta_{t+33} \eta_{t+34}\right)\left(\left(\left(\left(\left(\eta_{t+35} \eta_{t+36}\right) \eta_{t+37}\right) \eta_{t+38}\right) \eta_{t+39}\right) \eta_{t+40}\right)\right)\right)\right)\right)
\end{aligned}
$$

В силу свободы кольца $F \mathfrak{J}$ это равенство равносильно одной из следующих двух систем 
равенств:

$$
\begin{aligned}
& w_{l_{1}}\left(e_{1}, e_{2}\right) \cdots w_{l_{0}}\left(e_{1}, e_{2}\right)=\left(\left(\cdots \left(\left(\left(\left(\left(\left(\eta_{1} \eta_{2}\right) \eta_{3}\right) \eta_{4}\right)\left(\left(\left(\left(\eta_{5} \eta_{6}\right) \eta_{7}\right) \eta_{8}\right) \eta_{9}\right)\right)\right.\right.\right.\right. \\
& \left.\left.\left.\left.\quad\left(\left(\left(\left(\eta_{10} \eta_{11}\right) \eta_{12}\right) \eta_{13}\right) \eta_{14}\right)\left(\left(\left(\left(\left(\eta_{15} \eta_{16}\right) \eta_{17}\right) \eta_{18}\right) \eta_{19} \eta_{20}\right)\right)\right) \eta_{21}\right) \cdots\right) \eta_{t+20}\right) \\
& w_{l_{r_{0}+1}}\left(e_{1}, e_{2}\right)=\left(\left(\left(\left(\eta_{t+21} \eta_{t+22}\right) \eta_{t+23}\right) \eta_{t+24}\right)\left(\left(\left(\left(\eta_{t+25} \eta_{t+26}\right) \eta_{t+27}\right) \eta_{t+28}\right) \eta_{t+29}\right)\right) \\
& \left.\quad\left(\left(\left(\left(\left(\eta_{t+30} \eta_{t+31}\right) \eta_{t+32}\right) \eta_{t+33} \eta_{t+34}\right)\left(\left(\left(\left(\left(\eta_{t+35} \eta_{t+36}\right) \eta_{t+37}\right) \eta_{t+38}\right) \eta_{t+39}\right) \eta_{t+40}\right)\right)\right)\right) \\
& w_{l_{1}}\left(e_{1}, e_{2}\right) \cdots w_{l_{0}}\left(e_{1}, e_{2}\right) \\
& \quad=\left(\left(\left(\left(\left(\eta_{t+21} \eta_{t+22}\right) \eta_{t+23}\right) \eta_{t+24}\right)\left(\left(\left(\left(\eta_{t+25} \eta_{t+26}\right) \eta_{t+27}\right) \eta_{t+28}\right) \eta_{t+29}\right)\right)\right. \\
& \left.\quad\left(\left(\left(\left(\left(\eta_{t+30} \eta_{t+31}\right) \eta_{t+32}\right) \eta_{t+33} \eta_{t+34}\right)\left(\left(\left(\left(\left(\eta_{t+35} \eta_{t+36}\right) \eta_{t+37}\right) \eta_{t+38}\right) \eta_{t+39}\right) \eta_{t+40}\right)\right)\right)\right) \\
& w_{l_{r_{0}+1}}\left(e_{1}, e_{2}\right)=\left(\left(\cdots \left(\left(\left(\left(\left(\eta_{1} \eta_{2}\right) \eta_{3}\right) \eta_{4}\right)\left(\left(\left(\left(\eta_{5} \eta_{6}\right) \eta_{7}\right) \eta_{8}\right) \eta_{9}\right)\right)\right.\right.\right. \\
& \left.\left.\left.\left.\quad\left(\left(\left(\left(\eta_{10} \eta_{11}\right) \eta_{12}\right) \eta_{13}\right) \eta_{14}\right)\left(\left(\left(\left(\left(\eta_{15} \eta_{16}\right) \eta_{17}\right) \eta_{18}\right) \eta_{19} \eta_{20}\right)\right)\right) \eta_{21}\right) \cdots\right) \eta_{t+20}\right)
\end{aligned}
$$

Рассмотрим систему (30). Из второго равенства системы (30) получаем

$$
\begin{gathered}
E\left(e_{1}, e_{2}\right) e_{2}^{s} E\left(e_{1}, e_{2}\right)=\left(\left(\left(\left(\left(\eta_{t+21} \eta_{t+22}\right) \eta_{t+23}\right) \eta_{t+24}\right)\left(\left(\left(\left(\eta_{t+25} \eta_{t+26}\right) \eta_{t+27}\right) \eta_{t+28}\right) \eta_{t+29}\right)\right)\right. \\
\left.\quad\left(\left(\left(\left(\left(\eta_{t+30} \eta_{t+31}\right) \eta_{t+32}\right) \eta_{t+33} \eta_{t+34}\right)\left(\left(\left(\left(\left(\eta_{t+35} \eta_{t+36}\right) \eta_{t+37}\right) \eta_{t+38}\right) \eta_{t+39}\right) \eta_{t+40}\right)\right)\right)\right)
\end{gathered}
$$

Отсюда вытекает, что

$$
\begin{gathered}
E\left(e_{1}, e_{2}\right) e_{2}^{s}=\left(\left(\left(\left(\eta_{t+21} \eta_{t+22}\right) \eta_{t+23}\right) \eta_{t+24}\right)\left(\left(\left(\left(\eta_{t+25} \eta_{t+26}\right) \eta_{t+27}\right) \eta_{t+28}\right) \eta_{t+29}\right)\right) \\
E\left(e_{1}, e_{2}\right)=\left(\left(\left(\left(\eta_{t+30} \eta_{t+31}\right) \eta_{t+32}\right) \eta_{t+33} \eta_{t+34}\right)\right. \\
\left.\left.\left.\quad\left(\left(\left(\left(\eta_{t+35} \eta_{t+36}\right) \eta_{t+37}\right) \eta_{t+38}\right) \eta_{t+39}\right) \eta_{t+40}\right)\right)\right) \\
\left.E\left(e_{1}, e_{2}\right)=\left(\left(\left(\left(\eta_{t+21} \eta_{t+22}\right) \eta_{t+23}\right) \eta_{t+24}\right)\left(\left(\left(\left(\eta_{t+25} \eta_{t+26}\right) \eta_{t+27}\right) \eta_{t+28}\right) \eta_{t+29}\right)\right)\right) \\
E\left(e_{1}, e_{2}\right) e_{2}^{s}=\left(\left(\left(\left(\left(\eta_{t+30} \eta_{t+31}\right) \eta_{t+32}\right) \eta_{t+33} \eta_{t+34}\right)\right.\right. \\
\left.\left.\left.\quad\left(\left(\left(\left(\eta_{t+35} \eta_{t+36}\right) \eta_{t+37}\right) \eta_{t+38}\right) \eta_{t+39}\right) \eta_{t+40}\right)\right)\right)
\end{gathered}
$$

Из системы (32) получаем $e_{2}=\left(\left(\eta_{t+21} \eta_{t+22}\right) \eta_{t+23}\right) \eta_{t+24}$ или $e_{2}=\left(\left(\left(\eta_{t+25} \eta_{t+26}\right) \eta_{t+27}\right)\right.$ $\left.\eta_{t+28}\right) \eta_{t+29}$. Очевидно, что оба эти равенства ложны. Поэтому и система (32) ложна. Из системы (33) получаем $e_{2}=\left(\left(\left(\eta_{t+30} \eta_{t+31}\right) \eta_{t+32}\right) \eta_{t+33}\right) \eta_{t+34}$ или $e_{2}=\left(\left(\left(\eta_{t+35} \eta_{t+36}\right)\right.\right.$ $\left.\left.\left.\eta_{t+37}\right) \eta_{t+38}\right) \eta_{t+39}\right) \eta_{t+40}$. Так как оба эти равенства ложны, то ложна система (33), а значит, ложна и система (30). Теперь рассмотрим систему (31). Из второго равенства системы (31) получаем

$$
\begin{aligned}
E\left(e_{1}, e_{2}\right) e_{2}^{s} E\left(e_{1}, e_{2}\right)= & \left(\left(\cdots \left(\left(( ( ( ( \eta _ { 1 } \eta _ { 2 } ) \eta _ { 3 } ) \eta _ { 4 } ) ( ( ( ( \eta _ { 5 } \eta _ { 6 } ) \eta _ { 7 } ) \eta _ { 8 } ) \eta _ { 9 } ) ) \left(\left(\left(\left(\left(\eta_{10} \eta_{11}\right) \eta_{12}\right) \eta_{13} \eta_{14}\right)\right.\right.\right.\right.\right.\right. \\
& \left.\left.\left.\left.\left.\left.\left(\left(\left(\left(\left(\eta_{15} \eta_{16}\right) \eta_{17}\right) \eta_{18}\right) \eta_{19}\right) \eta_{20}\right)\right)\right) \eta_{21}\right) \cdots\right) \eta_{t+20}\right)\right) .
\end{aligned}
$$

Отсюда вытекает

$$
\begin{aligned}
E\left(e_{1}, e_{2}\right)= & \left(\left(\cdots \left(( ( ( ( \eta _ { 1 } \eta _ { 2 } ) \eta _ { 3 } ) \eta _ { 4 } ) ( ( ( ( \eta _ { 5 } \eta _ { 6 } ) \eta _ { 7 } ) \eta _ { 8 } ) \eta _ { 9 } ) ) \left(\left(\left(\left(\left(\eta_{10} \eta_{11}\right) \eta_{12}\right) \eta_{13} \eta_{14}\right)\right.\right.\right.\right.\right. \\
& \left.\left.\left.\left.\left.\left.\left(\left(\left(\left(\left(\eta_{15} \eta_{16}\right) \eta_{17}\right) \eta_{18}\right) \eta_{19}\right) \eta_{20}\right)\right)\right) \eta_{21}\right) \cdots\right) \eta_{t+19}\right)\right), \\
E\left(e_{1}, e_{2}\right) e_{2}^{s}=\eta_{t+20} & \\
E\left(e_{1}, e_{2}\right) e_{2}^{s}= & \left(\left(\cdots \left(\left(( ( ( ( \eta _ { 1 } \eta _ { 2 } ) \eta _ { 3 } ) \eta _ { 4 } ) ( ( ( ( \eta _ { 5 } \eta _ { 6 } ) \eta _ { 7 } ) \eta _ { 8 } ) \eta _ { 9 } ) ) \left(\left(\left(\left(\left(\eta_{10} \eta_{11}\right) \eta_{12}\right) \eta_{13} \eta_{14}\right)\right.\right.\right.\right.\right.\right. \\
& \left.\left.\left.\left.\left.\left.\left(\left(\left(\left(\left(\eta_{15} \eta_{16}\right) \eta_{17}\right) \eta_{18}\right) \eta_{19}\right) \eta_{20}\right)\right)\right) \eta_{21}\right) \cdots\right) \eta_{t+19}\right)\right), \\
E\left(e_{1}, e_{2}\right)=\eta_{t+20} &
\end{aligned}
$$

Очевидно, что первое равенство системы (34) неверно. Из леммы 5 вытекает ложность первого равенства системы (35). Следовательно, ложна и система (31). Лемма 4 доказана. 
Лемма 4 доказывает базу индукции леммы 3. Предположим, что равенство (2) не выполняется для любого $r_{2} \leqslant r_{0}$. Покажем, что равенство $(2)$ ложно и для $r_{2}=r_{0}+1$. В самом деле, из равенства (2) при $r_{1}>1$ получаем

$$
\begin{gathered}
w_{l_{1}}\left(e_{1}, e_{2}\right) \cdots w_{l_{r_{1}-1}}\left(e_{1}, e_{2}\right)=w_{p_{1}}\left(\eta_{1 p_{1}}, \eta_{2 p_{1}}\right) \cdots w_{p_{r_{0}}}\left(\eta_{1 p_{r_{0}}}, \eta_{2 p_{r_{0}}}\right) \\
w_{l_{r_{1}}}\left(e_{1}, e_{2}\right)=w_{p_{r_{0}+1}}\left(\eta_{1 p_{r_{0}+1}}, \eta_{2 p_{r_{0}+1}}\right) \\
w_{l_{1}}\left(e_{1}, e_{2}\right) \cdots w_{l_{r_{1}-1}}\left(e_{1}, e_{2}\right)=w_{p_{r_{0}+1}}\left(\eta_{1 p_{r_{0}+1}}, \eta_{2 p_{r_{0}+1}}\right) \\
w_{p_{1}}\left(\eta_{1 p_{1}}, \eta_{2 p_{1}}\right) \cdots w_{p_{r_{0}}}\left(\eta_{1 p_{r_{0}}}, \eta_{2 p_{r_{0}}}\right)=w_{l_{r_{1}}}\left(e_{1}, e_{2}\right)
\end{gathered}
$$

Рассмотрим систему (36). Допустим, что $\eta_{i}$ такое, что $\eta_{i} \neq e_{1}$ и $\eta_{i} \neq e_{2}$, содержится в $w_{p_{1}}\left(\eta_{1 p_{1}}, \eta_{2 p_{1}}\right) \cdots w_{p_{r_{0}}}\left(\eta_{1 p_{r_{0}}}, \eta_{2 p_{r_{0}}}\right)$. Тогда по предположению индукции первое равенство системы (36) ложно. Если же $\eta_{i}$ содержится в $w_{p_{r_{0}+1}}\left(\eta_{1 p_{r_{0}+1}}, \eta_{2 p_{r_{0}+1}}\right)$, то по предположению индукции ложно второе равенство системы (36). Следовательно, система (36) ложна. Система (37) рассматривается аналогично. Лемма 3 доказана.

Итак, в силу леммы 3 можно считать, что многочлен

$$
w_{i_{1}}(x, y) w_{i_{2}}(x, y) \cdots w_{i_{s}}(x, y)-w_{j_{1}}(x, y) w_{j_{2}}(x, y) \cdots w_{j_{t}}(x, y)
$$

имеет вид

$$
\begin{aligned}
& w_{i_{1}}\left(\alpha e_{1}+\beta e_{2}, \gamma e_{1}+\delta e_{2}\right) \cdots w_{i_{s}}\left(\alpha e_{1}+\beta e_{2}, \gamma e_{1}+\delta e_{2}\right) \\
& \quad-w_{j_{1}}\left(\alpha e_{1}+\beta e_{2}, \gamma e_{1}+\delta e_{2}\right) \cdots w_{j_{t}}\left(\alpha e_{1}+\beta e_{2}, \gamma e_{1}+\delta e_{2}\right) .
\end{aligned}
$$

Очевидно, что если $\alpha=\gamma=1, \beta=\delta=0$ или $\alpha=\gamma=0, \beta=\delta=1$, то многочлен (38) равен 0. Таким образом, нам осталось рассмотреть следующие случаи:

1) $\alpha=1, \beta=1, \gamma=1, \delta=0$;

2) $\alpha=1, \beta=0, \gamma=1, \delta=1$;

3) $\alpha=0, \beta=1, \gamma=0, \delta=0$;

4) $\alpha=0, \beta=0, \gamma=0, \delta=1$;

5) $\alpha=1, \beta=1, \gamma=0, \delta=1$;

6) $\alpha=0, \beta=1, \gamma=1, \delta=1$;

7) $\alpha=1, \beta=0, \gamma=0, \delta=0$;

8) $\alpha=0, \beta=0, \gamma=1, \delta=0$;

9) $\alpha=1, \beta=1, \gamma=1, \delta=1$;

10) $\alpha=0, \beta=0, \gamma=0, \delta=0$;

11) $\alpha=1, \beta=1, \gamma=0, \delta=0$;

12) $\alpha=1, \beta=0, \gamma=0, \delta=1$;

13) $\alpha=0, \beta=1, \gamma=1, \delta=0$;

14) $\alpha=0, \beta=0, \gamma=1, \delta=1$.

Предположим, что имеет место случай 1$)$. Тогда $x=e_{1}+e_{2}, y=e_{1}$. В силу дистрибутивности и тождества $x^{2}=0$ отсюда получаем, что

$$
\begin{aligned}
& w_{i_{1}}(x, y) w_{i_{2}}(x, y) \cdots w_{i_{s}}(x, y)-w_{j_{1}}(x, y) w_{j_{2}}(x, y) \cdots w_{j_{t}}(x, y) \\
& \quad=w_{i_{1}}\left(e_{2}, e_{1}\right) w_{i_{2}}\left(e_{2}, e_{1}\right) \cdots w_{i_{s}}\left(e_{2}, e_{1}\right)-w_{j_{1}}\left(e_{2}, e_{1}\right) w_{j_{2}}\left(e_{2}, e_{1}\right) \cdots w_{j_{t}}\left(e_{2}, e_{1}\right)
\end{aligned}
$$




\section{Лемма 8. Равенство вида}

$$
w_{i_{1}}\left(e_{2}, e_{1}\right) w_{i_{2}}\left(e_{2}, e_{1}\right) \cdots w_{i_{s}}\left(e_{2}, e_{1}\right)=w_{j_{1}}\left(e_{1}, e_{2}\right) w_{j_{2}}\left(e_{1}, e_{2}\right) \cdots w_{j_{t}}\left(e_{1}, e_{2}\right)
$$

в кольие $F_{\omega} \mathfrak{J}$ ложно.

ДокАЗАТЕльСТво проведем индукцией по числу $t$. Предположим, что $t=1$. Тогда равенство (39) имеет вид

$$
w_{i_{1}}\left(e_{2}, e_{1}\right) w_{i_{2}}\left(e_{2}, e_{1}\right) \cdots w_{i_{s}}\left(e_{2}, e_{1}\right)=w_{j_{1}}\left(e_{1}, e_{2}\right) .
$$

Лемма 9. Равенство (40) ложно.

ДокаЗАТЕльСтво. Допустим, что $s=1$. Тогда равенство (40) имеет вид $w_{i_{1}}\left(e_{2}, e_{1}\right)$ $=w_{j_{1}}\left(e_{1}, e_{2}\right)$. Отсюда вытекает

$$
\begin{aligned}
& E\left(e_{1}, e_{2}\right) e_{2}^{p}=E\left(e_{2}, e_{1}\right) e_{1}^{k}, \quad E\left(e_{1}, e_{2}\right)=E\left(e_{2}, e_{1}\right) ; \\
& E\left(e_{1}, e_{2}\right) e_{2}^{p}=E\left(e_{2}, e_{1}\right), \quad E\left(e_{1}, e_{2}\right)=E\left(e_{2}, e_{1}\right) e_{1}^{k} .
\end{aligned}
$$

Из первого равенства системы (41) получаем либо равенство слов разной длины, либо $e_{1}=e_{2}$. Следовательно, система (41) ложна. Из первого равенства системы (42) получаем $e_{2}=\left(\left(\left(e_{2} e_{1}\right) e_{1}\right) e_{1}\right)\left(\left(\left(\left(e_{2} e_{1}\right) e_{1}\right) e_{1}\right) e_{1}\right)$ или $e_{2}=\left(\left(\left(\left(e_{2} e_{1}\right) e_{1}\right) e_{1}\right) e_{1}\right)\left(\left(\left(\left(e_{2} e_{1}\right) e_{1}\right) e_{1}\right)\right.$ $\left.\left.e_{1}\right) e_{1}\right)$. Оба эти равенства ложны. Следовательно, при $s=1$ равенство (40) неверно.

Предположим, что утверждение леммы справедливо для всех $s \leqslant s_{0}$. Покажем, что оно верно и для $s=s_{0}+1$. Из равенства (40) при $s=s_{0}+1$ вытекает

$$
\begin{aligned}
& w_{i_{1}}\left(e_{2}, e_{1}\right) w_{i_{2}}\left(e_{2}, e_{1}\right) \cdots w_{i_{s_{0}}}\left(e_{2}, e_{1}\right)=E\left(e_{1}, e_{2}\right) e_{2}^{k}, \quad w_{i_{s_{0}+1}}\left(e_{2}, e_{1}\right)=E\left(e_{1}, e_{2}\right) \\
& w_{i_{1}}\left(e_{2}, e_{1}\right) w_{i_{2}}\left(e_{2}, e_{1}\right) \cdots w_{i_{s_{0}}}\left(e_{2}, e_{1}\right)=E\left(e_{1}, e_{2}\right), \quad w_{i_{s_{0}+1}}\left(e_{2}, e_{1}\right)=E\left(e_{1}, e_{2}\right) e_{2}^{k} .
\end{aligned}
$$

Из второго равенства системы (43) получаем, что должно вьполняться одно из следующих двух равенств:

$$
\begin{aligned}
& E\left(e_{2}, e_{1}\right)=\left(\left(\left(e_{1} e_{2}\right) e_{2}\right) e_{2}\right)\left(\left(\left(\left(e_{1} e_{2}\right) e_{2}\right) e_{2}\right) e_{2}\right), \\
& E\left(e_{2}, e_{1}\right)=\left(\left(\left(\left(e_{1} e_{2}\right) e_{2}\right) e_{2}\right) e_{2}\right)\left(\left(\left(\left(\left(e_{1} e_{2}\right) e_{2}\right) e_{2}\right) e_{2}\right) e_{2}\right) .
\end{aligned}
$$

Легко понять, что оба эти равенства ложны, так как длины слов, стоящих в правых и левых частях равенств, не равны. Следовательно, система (43) не вьполняется. Система (44) рассматривается аналогично. Лемма 9 доказана.

Лемма 9 обосновывает базу индукции для леммы 8 . Предположим теперь, что утверждение леммы 8 справедливо для всех $t \leqslant t_{0}$. Покажем, что лемма выполняется и для $t=t_{0}+1$. Равенство (39) при $t=t_{0}+1$ вьполняется тогда и только тогда, когда справедлива одна из следующих систем равенств:

$$
\begin{gathered}
w_{i_{1}}\left(e_{2}, e_{1}\right) w_{i_{2}}\left(e_{2}, e_{1}\right) \cdots w_{i_{s-1}}\left(e_{2}, e_{1}\right)=w_{j_{t_{0}+1}}\left(e_{1}, e_{2}\right) \\
w_{j_{1}}\left(e_{1}, e_{2}\right) w_{j_{2}}\left(e_{1}, e_{2}\right) \cdots w_{j_{t_{0}}}\left(e_{1}, e_{2}\right)=w_{i_{s}}\left(e_{2}, e_{1}\right) \\
w_{i_{1}}\left(e_{2}, e_{1}\right) w_{i_{2}}\left(e_{2}, e_{1}\right) \cdots \\
w_{i_{s-1}}\left(e_{2}, e_{1}\right)=w_{j_{1}}\left(e_{1}, e_{2}\right) w_{j_{2}}\left(e_{1}, e_{2}\right) \cdots w_{j_{0}}\left(e_{1}, e_{2}\right), \\
w_{i_{s}}\left(e_{2}, e_{1}\right)=w_{j_{t_{0}+1}}\left(e_{1}, e_{2}\right) .
\end{gathered}
$$


По предположению индукции все четыре равенства, входящие в системы (45) и (46), ложны. Лемма 8 доказана.

Из леммы 8 вытекает, что многочлен (38) входит в многочлен $f_{2}$. Легко проверить, что в случаях 3$), 4), 7)-11$ ) и 14 ) многочлен (38) равен 0 , а случаи 6 ) и 13) тривиально сводятся к случаю 1). Как нетрудно убедиться, случаи 2) и 5) сводятся к случаю 12). Рассмотрим случай 12). Так как $\alpha=\delta=1, \beta=\gamma=0$, многочлен (38) имеет вид

$$
w_{i_{1}}\left(e_{1}, e_{2}\right) w_{i_{2}}\left(e_{1}, e_{2}\right) \cdots w_{i_{s}}\left(e_{1}, e_{2}\right)-w_{j_{1}}\left(e_{1}, e_{2}\right) w_{j_{2}}\left(e_{1}, e_{2}\right) \cdots w_{j_{t}}\left(e_{1}, e_{2}\right) .
$$

Следовательно,

$$
\begin{aligned}
u_{11} h_{1} u_{12}= & u_{11}\left(w_{i_{1}}\left(e_{1}, e_{2}\right) w_{i_{2}}\left(e_{1}, e_{2}\right) \cdots w_{i_{s}}\left(e_{1}, e_{2}\right)\right. \\
& \left.-w_{j_{1}}\left(e_{1}, e_{2}\right) w_{j_{2}}\left(e_{1}, e_{2}\right) \cdots w_{j_{t}}\left(e_{1}, e_{2}\right)\right) u_{12} .
\end{aligned}
$$

Покажем, что если $u_{11}\left(w_{i_{1}}\left(e_{1}, e_{2}\right) w_{i_{2}}\left(e_{1}, e_{2}\right) \cdots w_{i_{s}}\left(e_{1}, e_{2}\right)\right) u_{12}$ является одночленом многочлена $f_{1}$, то и слово $u_{11}\left(w_{j_{1}}\left(e_{1}, e_{2}\right) w_{j_{2}}\left(e_{1}, e_{2}\right) \cdots w_{j_{t}}\left(e_{1}, e_{2}\right)\right) u_{12}$ является одночленом многочлена $f_{1}$. Предположим, что

$$
u_{11}\left(w_{i_{1}}\left(e_{1}, e_{2}\right) w_{i_{2}}\left(e_{1}, e_{2}\right) \cdots w_{i_{s}}\left(e_{1}, e_{2}\right)\right) u_{12}=w_{l_{1}}\left(e_{1}, e_{2}\right) w_{l_{2}}\left(e_{1}, e_{2}\right) \cdots w_{l_{k}}\left(e_{1}, e_{2}\right) .
$$

Тогда слово $w_{i_{1}}\left(e_{1}, e_{2}\right) w_{i_{2}}\left(e_{1}, e_{2}\right) \cdots w_{i_{s}}\left(e_{1}, e_{2}\right)$ должно быть подсловом слова $w_{l_{1}}\left(e_{1}\right.$, $\left.e_{2}\right) w_{l_{2}}\left(e_{1}, e_{2}\right) \cdots w_{l_{k}}\left(e_{1}, e_{2}\right)$. Поэтому в кольце $F_{\omega} \mathfrak{J}$ вьполняется равенство

$$
w_{i_{1}}\left(e_{1}, e_{2}\right) w_{i_{2}}\left(e_{1}, e_{2}\right) \cdots w_{i_{s}}\left(e_{1}, e_{2}\right)=w_{l_{1}}\left(e_{1}, e_{2}\right) w_{l_{2}}\left(e_{1}, e_{2}\right) \cdots w_{l_{k-p}}\left(e_{1}, e_{2}\right)
$$

или существует $r$ такое, что $w_{i_{1}}\left(e_{1}, e_{2}\right) w_{i_{2}}\left(e_{1}, e_{2}\right) \cdots w_{i_{s}}\left(e_{1}, e_{2}\right)$ является подсловом слова $w_{l_{r}}\left(e_{1}, e_{2}\right)$. Заметим, что, поскольку выполняется равенство $(47)$, слово $u_{11}\left(w_{j_{1}}\left(e_{1}\right.\right.$, $\left.\left.e_{2}\right) w_{j_{2}}\left(e_{1}, e_{2}\right) \cdots w_{j_{t}}\left(e_{1}, e_{2}\right)\right) u_{12}$ является одночленом многочлена $f_{1}$ и имеет вид $w_{j_{1}}\left(e_{1}\right.$, $\left.e_{2}\right) w_{j_{2}}\left(e_{1}, e_{2}\right) \cdots w_{j_{t}}\left(e_{1}, e_{2}\right) w_{l_{k-p+1}}\left(e_{1}, e_{2}\right) \cdots w_{l_{k}}\left(e_{1}, e_{2}\right)$. Предположим теперь, что $w_{i_{1}}\left(e_{1}, e_{2}\right) w_{i_{2}}\left(e_{1}, e_{2}\right) \cdots w_{i_{s}}\left(e_{1}, e_{2}\right)$ является подсловом слова $w_{l_{r}}\left(e_{1}, e_{2}\right)$. Тогда это слово должно быть равно одному из следующих пяти слов:

$$
e_{1} e_{2}^{i}, i \in\{0,1, \ldots, 5\}, \quad\left(e_{1} e_{2}^{3}\right)\left(e_{1} e_{2}^{4}\right), \quad\left(e_{1} e_{2}^{4}\right)\left(e_{1} e_{2}^{5}\right), \quad E\left(e_{1}, e_{2}\right), \quad E\left(e_{1}, e_{2}\right) e_{2}^{q} .
$$

Заметим, что длины первых четырех слов не превосходят 20 , а длина слова $w_{i_{1}}\left(e_{1}, e_{2}\right)$ $w_{i_{2}}\left(e_{1}, e_{2}\right) \cdots w_{i_{s}}\left(e_{1}, e_{2}\right)$ больше 40 . Таким образом, в кольце $F_{\omega} \mathfrak{J}$ должно вьполняться равенство вида $w_{i_{1}}\left(e_{1}, e_{2}\right) w_{i_{2}}\left(e_{1}, e_{2}\right) \cdots w_{i_{s}}\left(e_{1}, e_{2}\right)=E\left(e_{1}, e_{2}\right) e_{2}^{q}$. Из этого равенства очевидньп образом вытекает одно из следующих равенств:

$$
w_{i_{1}}\left(e_{1}, e_{2}\right) w_{i_{2}}\left(e_{1}, e_{2}\right) \cdots w_{i_{s-1}}\left(e_{1}, e_{2}\right)=e_{2}, \quad w_{i_{s}}\left(e_{1}, e_{2}\right)=e_{2} .
$$

Так как оба эти равенства ложны, то $w_{i_{1}}\left(e_{1}, e_{2}\right) w_{i_{2}}\left(e_{1}, e_{2}\right) \cdots w_{i_{s}}\left(e_{1}, e_{2}\right)$ не является подсловом слова $w_{l_{r}}\left(e_{1}, e_{2}\right)$, а значит, слово $u_{11}\left(w_{i_{1}}\left(e_{1}, e_{2}\right) w_{i_{2}}\left(e_{1}, e_{2}\right) \cdots w_{i_{s}}\left(e_{1}, e_{2}\right)\right) u_{12}$ является одночленом многочлена $f_{1}$ тогда и только тогда, когда слово $u_{11}\left(w_{j_{1}}\left(e_{1}, e_{2}\right)\right.$ $\left.w_{j_{2}}\left(e_{1}, e_{2}\right) \cdots w_{j_{t}}\left(e_{1}, e_{2}\right)\right) u_{12}$ является одночленом этого многочлена.

Итак, мы показали, что если многочлен $h_{1}$ соответствует тождеству многообразия $\mathfrak{M}$, то многочлен $u_{11} h_{1} u_{12}$ представим в виде суммы $f_{1}+f_{2}$, где $f_{1}$ и $f_{2}$ - многочлены с указанными вьше свойствами.

Случай многообразия $\mathfrak{B}$. Так как многочлен $h_{1}$ получен из тождества многообразия $\mathfrak{B}$, он имеет вид $z w_{1}(x, y) w_{2}(x, y)-z w_{2}(x, y) w_{1}(x, y)$. Без ограничения общности можно считать, что в многочлен $\sum_{i, j} u_{i 1} h_{j} u_{i 2}$ входят только образующие $e_{1}$ и $e_{2}$. 
Кроме того, в силу дистрибутивности и линейности вхождения $z$ в многочлен $u_{11} h_{1} u_{12}$ можно считать, что $z$ - одночлен. Представим $x$ и $y$ в виде $\alpha e_{1}+\beta e_{2}+x^{\star}\left(e_{1}, e_{2}\right)$ и $\gamma e_{1}+\delta e_{2}+y^{\star}\left(e_{1}, e_{2}\right)$, где $\alpha, \beta, \gamma, \delta \in\{0,1\}, x^{\star}\left(e_{1}, e_{2}\right), y^{\star}\left(e_{1}, e_{2}\right)$ - многочлены от $e_{1}, e_{2}$, нижняя степень которых не меньше 2 . Тогда

$$
\begin{aligned}
& z w_{1}(x, y) w_{2}(x, y)-z w_{2}(x, y) w_{1}(x, y) \\
& =z w_{1}\left(\alpha e_{1}+\beta e_{2}+x^{\star}\left(e_{1}, e_{2}\right), \gamma e_{1}+\delta e_{2}+y^{\star}\left(e_{1}, e_{2}\right)\right) \\
& w_{2}\left(\alpha e_{1}+\beta e_{2}+x^{\star}\left(e_{1}, e_{2}\right), \gamma e_{1}+\delta e_{2}+y^{\star}\left(e_{1}, e_{2}\right)\right) \\
& -z w_{2}\left(\alpha e_{1}+\beta e_{2}+x^{\star}\left(e_{1}, e_{2}\right), \gamma e_{1}+\delta e_{2}+y^{\star}\left(e_{1}, e_{2}\right)\right) \\
& w_{1}\left(\alpha e_{1}+\beta e_{2}+x^{\star}\left(e_{1}, e_{2}\right), \gamma e_{1}+\delta e_{2}+y^{\star}\left(e_{1}, e_{2}\right)\right) \\
& =z w_{1}\left(\alpha e_{1}+\beta e_{2}, \gamma e_{1}+\delta e_{2}\right) w_{2}\left(\alpha e_{1}+\beta e_{2}, \gamma e_{1}+\delta e_{2}\right) \\
& -z w_{2}\left(\alpha e_{1}+\beta e_{2}, \gamma e_{1}+\delta e_{2}\right) w_{1}\left(\alpha e_{1}+\beta e_{2}, \gamma e_{1}+\delta e_{2}\right)+U\left(e_{1}, e_{2}\right),
\end{aligned}
$$

где $U\left(e_{1}, e_{2}\right)$ - многочлен, в каждьй одночлен которого в качестве подслова входит одночлен многочлена $x^{\star}\left(e_{1}, e_{2}\right)$ или $y^{\star}\left(e_{1}, e_{2}\right)$. Покажем, что каждьй одночлен многочлена $U\left(e_{1}, e_{2}\right)$ является одночленом многочлена $f_{2}$. Допустим противное. Тогда в кольце $F_{\omega} \mathfrak{J}$ должно выполняться равенство следующего вида:

$$
w_{i_{1}}\left(e_{1}, e_{2}\right) w_{i_{2}}\left(e_{1}, e_{2}\right) \cdots w_{i_{k}}\left(e_{1}, e_{2}\right)=z w_{1}\left(\vec{\eta}_{1}, \vec{\eta}_{2}\right) w_{2}\left(\vec{\eta}_{3}, \vec{\eta}_{4}\right)
$$

причем для некоторого $j \eta_{j} \neq e_{1}$ и $\eta_{j} \neq e_{2}$.

Лемма 10. Равенство (48) ложно.

ДокАЗАТЕЛЬСтво. Предположим, что равенство (48) имеет место. Пусть $k=1$. Тогда равенство $(48)$ имеет вид $w_{i_{1}}\left(e_{1}, e_{2}\right)=z w_{1}\left(\vec{\eta}_{1}, \vec{\eta}_{2}\right) w_{2}\left(\vec{\eta}_{3}, \vec{\eta}_{4}\right)$. Отсюда, как нетрудно убедиться, вытекает одна из следующих систем равенств:

$$
\begin{aligned}
& E\left(e_{1}, e_{2}\right) e_{2}^{s}=z w_{1}\left(\vec{\eta}_{1}, \vec{\eta}_{2}\right), \quad E\left(e_{1}, e_{2}\right)=w_{2}\left(\vec{\eta}_{3}, \vec{\eta}_{4}\right) \\
& E\left(e_{1}, e_{2}\right) e_{2}^{s}=w_{2}\left(\vec{\eta}_{3}, \vec{\eta}_{4}\right), \quad E\left(e_{1}, e_{2}\right)=z w_{1}\left(\vec{\eta}_{1}, \vec{\eta}_{2}\right) .
\end{aligned}
$$

Рассмотрим систему (49). Легко понять, что длина слова $w_{2}\left(\overrightarrow{\eta_{3}}, \overrightarrow{\eta_{4}}\right)$ больше 40 , а длина слова $E\left(e_{1}, e_{2}\right)$ равна 20 . Следовательно, второе равенство системы $(49)$ не вьполняется, а значит, система (49) ложна. Из системы (50) вытекает одно из следующих равенств:

$$
\begin{aligned}
e_{2}= & \left(\left(\cdots \left(( ( ( ( ( \eta _ { 1 } \eta _ { 2 } ) \eta _ { 3 } ) \eta _ { 4 } ) ) ( ( ( ( \eta _ { 5 } \eta _ { 6 } ) \eta _ { 7 } ) \eta _ { 8 } ) \eta _ { 9 } ) ) \left(\left(\left(\left(\left(\eta_{10} \eta_{11}\right) \eta_{12}\right) \eta_{13} \eta_{14}\right)\right.\right.\right.\right.\right. \\
& \left.\left.\left.\left.\left.\left.\left(\left(\left(\left(\left(\eta_{15} \eta_{16}\right) \eta_{17}\right) \eta_{18}\right) \eta_{19}\right) \eta_{20}\right)\right)\right) \eta_{21}\right) \cdots\right) \eta_{t+20}\right)\right) \\
e_{2}= & \left(\left(\left(\left(\left(\eta_{t+21} \eta_{t+22}\right) \eta_{t+23}\right) \eta_{t+24}\right)\left(\left(\left(\left(\eta_{t+25} \eta_{t+26}\right) \eta_{t+27}\right) \eta_{t+28}\right) \eta_{t+29}\right)\right)\right. \\
& \left.\left(\left(\left(\left(\left(\eta_{t+30} \eta_{t+31}\right) \eta_{t+32}\right) \eta_{t+33} \eta_{t+34}\right)\left(\left(\left(\left(\left(\eta_{t+35} \eta_{t+36}\right) \eta_{t+37}\right) \eta_{t+38}\right) \eta_{t+39}\right) \eta_{t+40}\right)\right)\right)\right) .
\end{aligned}
$$

Очевидно, что оба эти равенства ложны. Следовательно, ложна и система (50). Таким образом, при $k=1$ равенство (48) ложно. Пусть $k=2$. Тогда равенство (48) имеет вид $w_{i_{1}}\left(e_{1}, e_{2}\right) w_{i_{2}}\left(e_{1}, e_{2}\right)=z w_{1}\left(\vec{\eta}_{1}, \vec{\eta}_{2}\right) w_{2}\left(\vec{\eta}_{3}, \vec{\eta}_{4}\right)$. Отсюда, как нетрудно убедиться, вытекает одна из следующих систем равенств:

$$
\begin{aligned}
& w_{i_{1}}\left(e_{1}, e_{2}\right)=z w_{1}\left(\vec{\eta}_{1}, \vec{\eta}_{2}\right), \quad w_{i_{2}}\left(e_{1}, e_{2}\right)=w_{2}\left(\vec{\eta}_{3}, \vec{\eta}_{4}\right) \\
& w_{i_{1}}\left(e_{1}, e_{2}\right)=w_{2}\left(\vec{\eta}_{3}, \vec{\eta}_{4}\right), \quad w_{i_{2}}\left(e_{1}, e_{2}\right)=z w_{1}\left(\vec{\eta}_{1}, \vec{\eta}_{2}\right)
\end{aligned}
$$


Рассмотрим систему (51). В силу леммы 3 из равенства $w_{i_{2}}\left(e_{1}, e_{2}\right)=w_{2}\left(\vec{\eta}_{3}, \vec{\eta}_{4}\right)$ вытекает, что если для любого $j \eta_{j}$ входит в $w_{2}$, то $\eta_{j}=e_{1}$ или $\eta_{j}=e_{2}$. Следовательно, существует $j$ такое, что $\eta_{j} \neq e_{1}$ и $\eta_{j} \neq e_{2}$, и, кроме того, $\eta_{j}$ - подслово слова $w_{1}$. Из равенства $w_{i_{1}}\left(e_{1}, e_{2}\right)=z w_{1}\left(\vec{\eta}_{3}, \vec{\eta}_{4}\right)$ вытекает одно из следующих равенств:

$$
E\left(e_{1}, e_{2}\right) e_{2}^{s}=w_{1}\left(\vec{\eta}_{1}, \vec{\eta}_{2}\right), \quad E\left(e_{1}, e_{2}\right)=w_{1}\left(\vec{\eta}_{1}, \vec{\eta}_{2}\right)
$$

Поскольку длина слова $w_{1}\left(\vec{\eta}_{1}, \vec{\eta}_{2}\right)$ больше 40 , а длина слова $E\left(e_{1}, e_{2}\right)$ равна 20 , второе равенство ложно. Из первого равенства вытекает одно из следующих двух равенств:

$$
\begin{aligned}
e_{2}= & \left(\left(\cdots \left(( ( ( ( ( \eta _ { 1 } \eta _ { 2 } ) \eta _ { 3 } ) \eta _ { 4 } ) ) ( ( ( ( \eta _ { 5 } \eta _ { 6 } ) \eta _ { 7 } ) \eta _ { 8 } ) \eta _ { 9 } ) ) \left(\left(\left(\left(\left(\eta_{10} \eta_{11}\right) \eta_{12}\right) \eta_{13} \eta_{14}\right)\right.\right.\right.\right.\right. \\
& \left.\left.\left.\left.\left.\left.\left(\left(\left(\left(\left(\eta_{15} \eta_{16}\right) \eta_{17}\right) \eta_{18}\right) \eta_{19}\right) \eta_{20}\right)\right)\right) \eta_{21}\right) \cdots\right) \eta_{t+20}\right)\right) \\
e_{2}= & \left(\left(\left(\left(\left(\eta_{t+21} \eta_{t+22}\right) \eta_{t+23}\right) \eta_{t+24}\right)\left(\left(\left(\left(\eta_{t+25} \eta_{t+26}\right) \eta_{t+27}\right) \eta_{t+28}\right) \eta_{t+29}\right)\right)\right. \\
& \left.\left(\left(\left(\left(\left(\eta_{t+30} \eta_{t+31}\right) \eta_{t+32}\right) \eta_{t+33} \eta_{t+34}\right)\left(\left(\left(\left(\left(\eta_{t+35} \eta_{t+36}\right) \eta_{t+37}\right) \eta_{t+38}\right) \eta_{t+39}\right) \eta_{t+40}\right)\right)\right)\right) .
\end{aligned}
$$

Так как оба эти равенства ложны, то ложно и равенство $w_{i_{1}}\left(e_{1}, e_{2}\right)=z w_{1}\left(\vec{\eta}_{3}, \vec{\eta}_{4}\right)$. Следовательно, система (51) ложна. Система (52) рассматривается аналогично. Поэтому при $k=2$ равенство (48) не вьполняется. Допустим теперь, что $k \geqslant 3$. Тогда из равенства (48) вытекает одна из следующих систем равенств:

$$
\begin{aligned}
& w_{i_{1}}\left(e_{1}, e_{2}\right) \cdots w_{i_{k-1}}\left(e_{1}, e_{2}\right)=z w_{1}\left(\vec{\eta}_{1}, \vec{\eta}_{2}\right), \quad w_{i_{k}}\left(e_{1}, e_{2}\right)=w_{2}\left(\vec{\eta}_{3}, \vec{\eta}_{4}\right) \\
& w_{i_{1}}\left(e_{1}, e_{2}\right) \cdots w_{i_{k-1}}\left(e_{1}, e_{2}\right)=w_{2}\left(\vec{\eta}_{3}, \vec{\eta}_{4}\right), \quad w_{i_{k}}\left(e_{1}, e_{2}\right)=z w_{1}\left(\vec{\eta}_{1}, \vec{\eta}_{2}\right) .
\end{aligned}
$$

В силу леммы 3 второе равенство системы (53) и первое равенство системы (54) ложны. Таким образом, для любого $k$ равенство (48) неверно. Лемма 10 доказана.

ЛЕмма 11. Если выполняется равенство

$$
w_{i_{1}}\left(e_{1}, e_{2}\right) \cdots w_{i_{k}}\left(e_{1}, e_{2}\right)=z w_{1}(x, y) w_{2}(x, y),
$$

mo $w_{j_{1}}\left(e_{1}, e_{2}\right) \cdots w_{j_{p}}\left(e_{1}, e_{2}\right)=z$.

ДокАЗАТЕЛЬСТво. Из равенства (55) при $k>2$ получаем

$$
w_{i_{1}}\left(e_{1}, e_{2}\right) \cdots w_{i_{k-1}}\left(e_{1}, e_{2}\right)=z w_{1}(x, y), \quad w_{i_{k}}\left(e_{1}, e_{2}\right)=w_{2}(x, y)
$$

или

$$
w_{i_{1}}\left(e_{1}, e_{2}\right) \cdots w_{i_{k-1}}\left(e_{1}, e_{2}\right)=w_{2}(x, y), \quad w_{i_{k}}\left(e_{1}, e_{2}\right)=z w_{1}(x, y) .
$$

Рассмотрим систему (56). Из первого равенства этой системы получаем

$$
w_{i_{1}}\left(e_{1}, e_{2}\right) \cdots w_{i_{k-2}}\left(e_{1}, e_{2}\right)=z, \quad w_{i_{k-1}}\left(e_{1}, e_{2}\right)=w_{1}(x, y)
$$

или

$$
w_{i_{1}}\left(e_{1}, e_{2}\right) \cdots w_{i_{k-2}}\left(e_{1}, e_{2}\right)=w_{1}(x, y), \quad w_{i_{k-1}}\left(e_{1}, e_{2}\right)=z
$$

что и требовалось доказать. Допустим, что вьполняется система (57). Тогда из первого равенства этой системы получаем

$$
\begin{aligned}
& E\left(e_{1}, e_{2}\right) e_{2}^{s}=z, \quad E\left(e_{1}, e_{2}\right)=w_{1}(x, y) \\
& E\left(e_{1}, e_{2}\right) e_{2}^{s}=w_{1}(x, y), \quad E\left(e_{1}, e_{2}\right)=z
\end{aligned}
$$


Очевидно, что второе равенство системы (58) неверно, следовательно, она ложна. Из первого равенства системы (59) получаем одно из следующих двух равенств:

$$
\begin{gathered}
\left(\left(\cdots \left(\left(( ( ( ( x _ { 1 } y _ { 1 } ) y _ { 2 } ) y _ { 3 } ) ( ( ( ( x _ { 2 } y _ { 4 } ) y _ { 5 } ) y _ { 6 } ) y _ { 7 } ) ) \left(\left(\left(\left(\left(x_{3} y_{8}\right) y_{9}\right) y_{10}\right) y_{11}\right)\right.\right.\right.\right.\right. \\
\left.\left.\left.\left.\left.\left(\left(\left(\left(\left(x_{4} y_{12}\right) y_{13}\right) y_{14}\right) y_{15}\right) y_{16}\right)\right)\right) y_{17}\right) \cdots\right) y_{t+17}\right)=e_{2} \\
\left(\left(\left(\left(\left(x_{5} y_{t+18}\right) y_{t+19}\right) y_{t+20}\right)\left(\left(\left(\left(x_{6} y_{t+21}\right) y_{t+22}\right) y_{t+23}\right) y_{t+24}\right)\right)\right. \\
\left.\left(\left(\left(\left(\left(x_{7} y_{t+25}\right) y_{t+26}\right) y_{t+27}\right) y_{t+28}\right)\left(\left(\left(\left(\left(x_{8} y_{t+29}\right) y_{t+30}\right) y_{t+31}\right) y_{t+32}\right) y_{t+33}\right)\right)\right)=e_{2} .
\end{gathered}
$$

Так как оба эти равенства ложны, то ложна система (59), а значит, и система (57). Таким образом, при $k>2$ равенство (55) не выполняется. Пусть $k=2$. Тогда из равенства (55) получаем

$$
\begin{array}{ll}
w_{i_{1}}\left(e_{1}, e_{2}\right)=z w_{1}(x, y), & w_{i_{2}}\left(e_{1}, e_{2}\right)=w_{2}(x, y) \\
w_{i_{2}}\left(e_{1}, e_{2}\right)=z w_{1}(x, y), & w_{i_{1}}\left(e_{1}, e_{2}\right)=w_{2}(x, y)
\end{array}
$$

При рассмотрении системы (57) мы показали, что равенства $w_{i}\left(e_{1}, e_{2}\right)=z w_{j}(x, y)$ ложны. Следовательно, при $k=2$ равенство (55) неверно.

Пусть теперь $k=1$. Тогда обязательно должно выполняться одно из следующих равенств: $E\left(e_{1}, e_{2}\right)=z w_{1}(x, y), E\left(e_{1}, e_{2}\right)=w_{2}(x, y)$. Так как длины слов $z w_{1}(x, y)$ и $w_{2}(x, y)$ больше 40 , а длина слова $E\left(e_{1}, e_{2}\right)$ равна 20 , оба эти равенства ложны. Следовательно, при $k=1$ равенство (55) неверно. Лемма 11 доказана.

Итак, для завершения доказательства в случае многообразия $\mathfrak{B}$ ввиду лемм 10 и 11 достаточно показать, что многочлен

$$
\begin{aligned}
& u_{11}\left(z w_{1}\left(\alpha e_{1}+\beta e_{2}, \gamma e_{1}+\delta e_{2}\right) w_{2}\left(\alpha e_{1}+\beta e_{2}, \gamma e_{1}+\delta e_{2}\right)\right. \\
& \left.\quad-z w_{2}\left(\alpha e_{1}+\beta e_{2}, \gamma e_{1}+\delta e_{2}\right) w_{1}\left(\alpha e_{1}+\beta e_{2}, \gamma e_{1}+\delta e_{2}\right)\right) u_{12}
\end{aligned}
$$

при $z=w_{i_{1}}\left(e_{1}, e_{2}\right) \cdots w_{i_{k}}\left(e_{1}, e_{2}\right)$ представим в виде суммы $f_{1}+f_{2}$, где $f_{1}$ и $f_{2}-$ многочлены с указанными вьше свойствами. Проверка этого факта совершенно аналогична рассуждениям, проведенньм при рассмотрении многообразия $\mathfrak{M}$. Лемма 2 доказана.

В силу свободы кольца $F_{\omega} \mathfrak{J}$ из равенства $\mathfrak{F}\left(e_{1}, e_{2}\right)=f_{1}+f_{2}$ очевидным образом вытекает, что $\mathfrak{F}\left(e_{1}, e_{2}\right)=f_{1}, f_{2}=0$. Индукцией по числу одночленов в многочлене $f_{1}$ покажем, что если в кольце $\mathfrak{F}\left(e_{1}, e_{2}\right)=f_{1}+f_{2}$ выполняется равенство $\mathfrak{H}=f_{1}$, где $\mathfrak{H}-$ многочлен, полученньй из многочлена

$$
\begin{aligned}
& Q_{i}\left(e_{1}, e_{2}\right) A^{\xi_{1}}\left(e_{1}, e_{2}\right) B^{\xi_{2}}\left(e_{1}, e_{2}\right) C\left(e_{1}, e_{2}\right) D\left(e_{1}, e_{2}\right) \\
& \quad+Q_{j}\left(e_{1}, e_{2}\right) A^{\eta_{1}}\left(e_{1}, e_{2}\right) B^{\eta_{2}}\left(e_{1}, e_{2}\right) C\left(e_{1}, e_{2}\right) D\left(e_{1}, e_{2}\right)
\end{aligned}
$$

конечным числом перестановок вида

$$
\begin{array}{ll}
A\left(e_{1}, e_{2}\right) B\left(e_{1}, e_{2}\right) \leftrightarrow B\left(e_{1}, e_{2}\right) A\left(e_{1}, e_{2}\right), & A\left(e_{1}, e_{2}\right) D\left(e_{1}, e_{2}\right) \leftrightarrow D\left(e_{1}, e_{2}\right) A\left(e_{1}, e_{2}\right), \\
B\left(e_{1}, e_{2}\right) C\left(e_{1}, e_{2}\right) \leftrightarrow C\left(e_{1}, e_{2}\right) B\left(e_{1}, e_{2}\right), & C\left(e_{1}, e_{2}\right) D\left(e_{1}, e_{2}\right) \leftrightarrow D\left(e_{1}, e_{2}\right) C\left(e_{1}, e_{2}\right),
\end{array}
$$

то существует конечное вычисление на машине $\mathrm{M}$, при помоши которого она переходит либо из конфигурации $q_{i} \xi_{1} \xi_{2}$ в конфигурацию $q_{j} \eta_{1} \eta_{2}$, либо наоборот.

Допустим, что число одночленов в многочлене $f_{1}$ равно 0 . Тогда утверждение очевидно. Пусть утверждение справедливо для некоторого числа одночленов $d$. Покажем, что оно верно и для $d+1$. Предположим, что в кольце $F_{\omega} \mathfrak{J}$ вьполняется равенство 
$\mathfrak{H}=f_{1}$ и число одночленов в многочлене $f_{1}$ не превосходит $d+1$. Обозначим через $\mathfrak{H}_{1}$ и $\mathfrak{H}_{2}$ одночлены многочлена $\mathfrak{H}$. В силу того, что $\mathfrak{H}_{1}+\mathfrak{H}_{2}=\sum_{i, j} v_{i 1} h_{j} v_{i 2}-$ равенство в свободном кольце, получаем, что $\mathfrak{H}_{1}$ является одночленом многочлена $v_{i 1} h_{j} v_{i 2}$ для некоторых $i, j$. Без ограничения общности можно считать, что $i=j=1$. Так как $v_{11} h_{1} v_{12}=\mathfrak{G}_{1}+\mathfrak{G}_{2}$ и $\mathfrak{H}_{1}=\mathfrak{G}_{1}$, из равенства $\mathfrak{H}_{1}+\mathfrak{H}_{2}=\sum_{i, j} v_{i 1} h_{j} v_{i 2}$ вытекает равенство $\mathfrak{G}_{2}+\mathfrak{H}_{2}=\sum_{i>1, j} v_{i 1} h_{j} v_{i 2}$. По построению многочлена $f_{1}$, если $h_{1}$ соответствует тождеству многообразия $\mathfrak{M}$, то $\mathfrak{G}_{2}+\mathfrak{H}_{2}-$ многочлен, полученный при помощи преобразований (61) из многочлена

$$
\begin{aligned}
& Q_{i}\left(e_{1}, e_{2}\right) A^{\xi_{1}}\left(e_{1}, e_{2}\right) B^{\xi_{2}}\left(e_{1}, e_{2}\right) C\left(e_{1}, e_{2}\right) D\left(e_{1}, e_{2}\right) \\
& \quad+Q_{l}\left(e_{1}, e_{2}\right) A^{\tau_{1}}\left(e_{1}, e_{2}\right) B^{\tau_{2}}\left(e_{1}, e_{2}\right) C\left(e_{1}, e_{2}\right) D\left(e_{1}, e_{2}\right)
\end{aligned}
$$

причем существует команда машины $\mathrm{M}$, при помоши которой машина переходит из конфигурации $q_{i} \xi_{1} \xi_{2}$ в конфигурацию $q_{j} \tau_{1} \tau_{2}$, либо наоборот; если же $h_{1}$ соответствует тождеству многообразия $\mathfrak{B}$, то замена $\mathfrak{H}$ на $\mathfrak{G}_{2}+\mathfrak{H}_{2}$ есть применение одного из преобразований (61). Отсюда вытекает, в силу того что число одночленов в многочлене $\sum_{i>1, j} v_{i 1} h_{j} v_{i 2}$ меньше $d+1$, возможность применения индуктивного предположения к равенству $\mathfrak{G}_{2}+\mathfrak{H}_{2}=\sum_{i>1, j} v_{i 1} h_{j} v_{i 2}$. Таким образом, из равенства (60) вытекает существование вычисления на машине $\mathrm{M}$ между конфигурациями $q_{i} \xi_{1} \xi_{2}$ и $q_{j} \eta_{1} \eta_{2}$. Пусть $i=1, j=0, \xi_{1}=2^{n}, \xi_{2}=0, \eta_{1}=1, \eta_{2}=0$. Тогда из равенства (1) вытекает, что существует конечное вычисление на машине M, при помощи которого она переходит из конфигурации $q_{1} 2^{n} 0$ в конфигурацию $q_{0} 10$. По определению машины М это означает, что $n \in P$. Лемма 1 доказана.

Лемма 12. Eсли $n \in P$, mo $\mathfrak{X}=\mathfrak{F}(x, y)=0$.

ДокАЗАтЕльство. Покажем, что если машина М через конечное число тактов работы переходит из конфигурации $q_{i} \xi_{1} \xi_{2}$ в конфигурацию $q_{j} \eta_{1} \eta_{2}$, то в многообразии $\mathfrak{X}$ выполняется тождество

$$
Q_{i}(x, y) A^{\xi_{1}}(x, y) B^{\xi_{2}}(x, y) C(x, y) D(x, y)=Q_{j}(x, y) A^{\eta_{1}}(x, y) B^{\eta_{2}}(x, y) C(x, y) D(x, y) .
$$

Сделаем это индукцией по числу $r$ тактов работы машины М. Допустим, что $r=0$. Тогда утверждение очевидно. Пусть оно справедливо для некоторого $r$. Покажем, что оно верно и для $r+1$. Предположим, что на $(r+1)$-м шаге машина М, вьполняя команду $q_{j} \delta_{1} \delta_{2} \rightarrow q_{l} \varepsilon_{1} \varepsilon_{2}$, переходит из конфигурации $q_{j} \eta_{1} \eta_{2}$ в конфигурацию $q_{l}\left(\eta_{1}+\varepsilon_{1}\right)\left(\eta_{2}+\varepsilon_{2}\right)$. Следует разобрать четыре случая в зависимости от значений $\delta_{1}$ и $\delta_{2}$. Разберем только случай $\delta_{1}=1, \delta_{2}=0$, поскольку остальные рассматриваются аналогично. Так как $\delta_{1}=1, \delta_{2}=2$, то $\eta_{1}=0, \eta_{2} \neq 0$ и на $(r+1)$-м шаге вьполняется команда $q_{j} 10 \rightarrow q_{l} \varepsilon_{1} \varepsilon_{2}$. Следовательно, в многообразии $\mathfrak{X}$ выполняется тождество

$$
Q_{j}(x, y) B(x, y) C(x, y)=Q_{l}(x, y) A^{\varepsilon_{1}}(x, y) B^{\varepsilon_{2}+1}(x, y) C(x, y) .
$$

Отсюда вытекает, что

$$
\begin{aligned}
Q_{i} & (x, y) A^{\xi_{1}}(x, y) B^{\xi_{2}}(x, y) C(x, y) D(x, y)=Q_{j}(x, y) A^{\eta_{1}}(x, y) B^{\eta_{2}}(x, y) C(x, y) D(x, y) \\
& =Q_{j}(x, y) B^{\eta_{2}}(x, y) C(x, y) D(x, y)=Q_{j}(x, y) B(x, y) C(x, y) B^{\eta_{2}-1}(x, y) D(x, y) \\
& =Q_{l}(x, y) A^{\varepsilon_{1}}(x, y) B^{\varepsilon_{2}+1}(x, y) C(x, y) B^{\eta_{2}-1}(x, y) D(x, y) \\
& =Q_{l}(x, y) A^{\varepsilon_{1}}(x, y) B^{\varepsilon_{2}+\eta_{2}}(x, y) C(x, y) D(x, y) \\
& =Q_{l}(x, y) A^{\varepsilon_{1}+\eta_{1}}(x, y) B^{\varepsilon_{2}+\eta_{2}}(x, y) C(x, y) D(x, y) .
\end{aligned}
$$


Заметим, что первое равенство в данной цепочке равенств выполняется по предположению индукции, второе и шестое - согласно $\eta_{1}=0$, третье и пятое - в силу тождества $y B(x, y) C(x, y)=y C(x, y) B(x, y)$, четвертое - в силу тождества

$$
Q_{j}(x, y) B(x, y) C(x, y)=Q_{l}(x, y) A^{\varepsilon_{1}}(x, y) B^{\varepsilon_{2}+1}(x, y) C(x, y) .
$$

Допустим, что $n \in P$. Тогда по определению машины М существует конечное вычисление на машине $\mathrm{M}$, при помощи которого она переходит из конфигурации $q_{1} 2^{n} 0$ в конфигурацию $q_{0} 10$. Следовательно, в многообразии $\mathfrak{X}$ в силу сказанного вьше вьполняется тождество

$$
Q_{1}(x, y) A^{2^{n}}(x, y) C(x, y) D(x, y)=Q_{0}(x, y) A(x, y) C(x, y) D(x, y) .
$$

Лемма 12 доказана.

В силу лемм 1 и 12 получаем, что в многообразии $\mathfrak{X}$ тождество (62) выполняется тогда и только тогда, когда $n \in P$. Так как множество $P$ нерекурсивно, то теория $\forall \mathfrak{X}$ неразрешима. Теорема доказана.

Заметим, что все выкладки доказательства теоремы без малейших изменений можно перенести на случай, когда вместо многообразия, заданного тождествами $2 x=0$, $x^{2}=0$, рассматривается многообразие, заданное тождествами $2 x y=0, x^{2}=0$. Легко понять, что при этом мы построим пример конечно базируемого многообразия колец с неразрешимой эквациональной теорией, в котором свободное кольцо не является кольцом характеристики 2.

\section{СПИСОК ЦИТИРОВАННОЙ ЛИТЕРАТУРЫ}

[1] Chin L. H., Tarski A. Distributive and modular laws in the arithmetic of relation algebras // Univ. California Publ. Math. (N.S.). 1951. V. 1. P. 341-384.

[2] Tarski A. Some metalogical results concerning the calculus of relations // J. Symbolic Logic. 1953. V. 18. P. $188-189$.

[3] Tarski A., Givant S. A Formalization of Set Theory without Variables. Amer. Math. Soc. Colloq. Publ. V. 41. Providence (R.I.): Amer. Math. Soc., 1987.

[4] Kharlampovich O. G., Sapir M. V. Algorithmic problems in varieties // Internat. J. Algebra Comput. 1995. V. 5. № 4-5. P. 379-602.

[5] Мальцев А. И. Тождественные соотношения на многообразиях квазигрупп // Матем. сб. 1966. T. 69. № 1. C. 3-12.

[6] Коуровская тетрадь. 10-е изд. Новосибирск, 1986.

[7] Мурский В. Л.Несколько примеров многообразий полугрупп // Матем. заметки. 1968. T. 3. №6. C. $663-670$.

[8] Клейман Ю. Г. О тождествах в группах // Тр. ММО. 1982. Т. 44. С. 62-108.

[9] Попов В. Ю. Эквациональные теории многообразий метабелевых и коммутативных колец // Алгебра и логика. 1995. Т. 34. № 3. С. 347-361. 